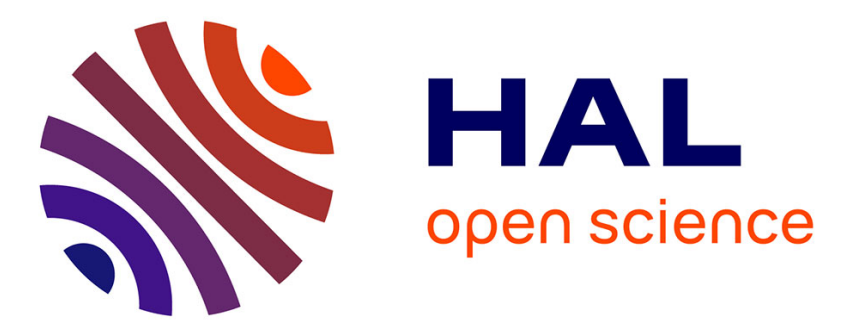

\title{
Generalized Khatri-Rao and Kronecker Space-Time Coding for MIMO Relay Systems with Closed-Form Semi-Blind Receivers
}

\author{
Walter Freitas, Gérard Favier, André L. F. de Almeida
}

\section{- To cite this version:}

Walter Freitas, Gérard Favier, André L. F. de Almeida. Generalized Khatri-Rao and Kronecker SpaceTime Coding for MIMO Relay Systems with Closed-Form Semi-Blind Receivers. Signal Processing, 2018, Signal Processing, 145, pp.304-316. hal-01766142

HAL Id: hal-01766142

https://hal.science/hal-01766142

Submitted on 13 Apr 2018

HAL is a multi-disciplinary open access archive for the deposit and dissemination of scientific research documents, whether they are published or not. The documents may come from teaching and research institutions in France or abroad, or from public or private research centers.
L'archive ouverte pluridisciplinaire HAL, est destinée au dépôt et à la diffusion de documents scientifiques de niveau recherche, publiés ou non, émanant des établissements d'enseignement et de recherche français ou étrangers, des laboratoires publics ou privés. 


\title{
Generalized Khatri-Rao and Kronecker Space-Time Coding for MIMO Relay Systems with Closed-Form Semi-Blind Receivers
}

\author{
Walter Freitas Jr. ${ }^{\mathrm{a}}$, Gérard Favier ${ }^{\mathrm{b}}$, André L. F. de Almeida ${ }^{\mathrm{c}}$ \\ ${ }^{a}$ Department of Teleinformatics Engineering, Federal University of Ceará, Fortaleza, Brazil \\ ${ }^{b}$ I3S Laboratory, University of Nice Sophia Antipolis, CNRS, Sophia Antipolis, France \\ ${ }^{c}$ Department of Teleinformatics Engineering, Federal University of Ceará, Fortaleza, Brazil
}

\begin{abstract}
In this paper, we propose two new space-time (ST) coding schemes for multiple-antenna signalling based on multiple Khatri-Rao and Kronecker products of symbol matrices, denoted MKRST and MKronST codings, respectively. These new coding schemes generalize the standard Khatri-Rao coding by introducing extra space/time diversities due to a mutual space-time spreading of the transmitted data streams, induced by Khatri-Rao and Kronecker products between symbol matrices. Parallel non-iterative decoding methods are proposed for estimating each symbol matrix. We consider a one-way two-hop multi-input multi-output (MIMO) relay system using the MKRST and MKronST codings at both the source and relay nodes, and three different relaying protocols, namely, amplify-and-forward (AF), decode-and-forward (DF) and estimate-and-forward (EF). For the DF and EF protocols, the channel and symbol estimation is split into two successive phases, one at the relay and the other one at the destination. Exploiting parallel factor (PARAFAC) models for the tensors of signals received at the relay and the destination, closed-form semi-blind receivers are derived for joint symbol and channel estimation. System parameters identifiability is also discussed, and the computational cost of the proposed receivers and decoders is detailed. Monte Carlo simulation results are provided to assess the performance of the proposed coding schemes, and semi-blind receivers.
\end{abstract}

Keywords: Khatri-Rao product, Kronecker product, PARAFAC, MIMO relaying, space time coding, semi-blind receivers.

\section{Introduction}

Currently, there are as many mobile subscriptions as people in the world. To support the tremendous increase of the resulting data traffic, one solution consists in moving closer the user equipments (UEs) to the base stations (BSs). That leads to what is called small-cells in cellular networks which will play an important role in future long-term evolution (LTE) networks. Another possibility is offered by cooperative infrastructures where relays help communications between source and destination nodes [1].

Email addresses: walter@gtel.ufc.br(Walter Freitas Jr.), favier@i3s.unice.fr(Gérard Favier), andre@gtel.ufc.br (André L. F. de Almeida)

${ }^{1}$ The authors would like to thank CAPES, COFECUB, FUNCAP, and CNPq for the financial support of this research. 
The basic result on cooperative communications is the seminal work [2] which established the capacity of certain relay channels. In [3], the benefits of cooperative communications were presented in terms of data rate, diversity and error performance in the context of code division multiple access (CDMA) systems. In [4], the authors discussed practical issues for implementation. In [5], low-complexity cooperative protocols were proposed and analyzed in the case of two cooperating terminals. Two relaying protocols are commonly used: AF, and DF. With the AF protocol, the relay retransmits the received signals without decoding, while DF involves decoding and re-encoding at the relay before forwarding the coded information to the destination. Note that recent works consider also non-orthogonal and orthogonal space-time block codes (OSTBC) in cooperative relaying systems [12] [13].

Since the pioneering work [6], the use of tensor decompositions has been widely studied for point-to-point wireless communication systems. The practical motivation for tensor modeling comes from the fact that one can simultaneously benefit from multiple (more than two) signal diversities, like space, time and frequency diversities, for instance. Therefore, tensor approaches allow to improve the reliability of wireless links while enabling a joint multiuser signal separation and channel estimation, under model uniqueness conditions more relaxed than those with conventional matrix-based solutions. Several tensor-based communication systems rely on the PARAFAC decomposition [7] of the received signals [6, 8, 9, 10], or on variants of this decomposition, such as, for instance, the block-PARAFAC models of [14, 15], and the more general framework of constrained PARAFAC models [11].

Such constrained PARAFAC models were proposed to design tensor-based MIMO communication systems, like the constrained factors (CONFAC) [16], PARATUCK-2 [17], PARATUCK-(2,4) [18], or still the generalized PARATUCK [19] decompositions. Note that in [20], three basic wireless communication systems are presented in an unified way by means of a constrained PARAFAC model which can be viewed as a block-CONFAC- $(2,3)$ model [11]. A nested PARAFAC model was also recently proposed for a double Khatri-Rao space-time-frequency coding [21].

In the context of cooperative wireless communications, a few results have been published on tensor-based receivers. Some works are dedicated to supervised channel estimation, i.e. with the use of training sequences, as in [22] and [23] - [24] for two-way and one-way two-hop relay systems, respectively. In [25] and [26], a scenario of one-way three-hop multi-relay system is considered, where multiple relay links are exploited at the receiver to estimate all the partial channels involved in the communication. However, all these works rely on supervised channel estimation methods, which can be bandwidth consuming, especially for moderate to large number of antennas. In [27] and [28], semi-blind receivers based on the PARAFAC model have been introduced for uplink multiuser cooperative communication systems, by assuming single-antenna relays in clustered propagation scenarios. In [29], a general scenario with $K$ relays assuming Khatri-Rao space-time coding at each relay is modeled by means of a generalized nested PARAFAC model which can be decomposed into $K+1$ third-order PARAFAC models for jointly estimating the information symbols and the individual channels, at the destination node. Recent works have proposed semi-blind receivers with joint symbol and channel estimation by considering multiple-antenna (MIMO) relays, which do not require training sequences. In this context, we can cite the PARAFAC-PARATUCK receiver [30] and the nested PARAFAC receiver [31, 32]. These works consider an amplify-and-forward (AF) relaying protocol.

In this paper, we first propose generalized Khatri-Rao and Kronecker ST codes for two-hop MIMO relay 
systems. This approach combines a simplified KRST coding [8], i.e. without pre-coding, with extra time (and space) spreading introduced by multiple Khatri-Rao (and Kronecker) products of symbol matrices. The resulting ST codings are called multiple Khatri-Rao product-based space-time (MKRST) and multiple Kronecker product-based space-time (MKronST) codings. Using these MKRST and MKronST codings at the source and the relay, tensor models are derived for the signals received at the relay and the destination, and closed-form semi-blind receivers using rank-one matrix approximations by means of singular value decompositions (SVD)s are presented.

We consider three different relaying protocols in this work. More specifically, in addition to the usual AF protocol, we also consider DF and EF relaying protocols. With the AF protocol, we propose a semi-blind receiver that exploits two separate PARAFAC models to estimate the channel and symbol matrices at the destination only, whereas for DF and EF the joint channel and symbol estimation is split into two phases, one at the relay and the other one at the destination. Identifiability conditions and scaling ambiguity relations are derived for the PARAFAC models exploited by the proposed receivers, and their computational complexities are analyzed.

The main contributions of this paper can be summarized as follows:

- We propose two new space-time (ST) coding schemes based on multiple Khatri-Rao and Kronecker products of symbol matrices, denoted MKRST and MKronST, respectively. Compared to the standard Khatri-Rao coding [8], these new ST codings allow to introduce extra space/time diversities, thanks to a mutual space-time spreading of the transmitted data streams. A singular value decomposition (SVD)-based rank-one approximation is proposed to estimate in parallel each symbol matrix of the Khatri-Rao and Kronecker products, avoiding error propagation inherent to the iterative closed-form solution presented in the Appendix.

- We describe a one-way two-hop MIMO relaying system using the proposed ST codings both at the source and relay nodes. Three different relaying protocols are considered, namely AF, DF and EF. Then, PARAFAC tensor models are established for the signals received at the relay and the destination.

- Exploiting the algebraic structure of these PARAFAC models, we derive closed-form semi-blind receivers for jointly estimating the two-hop channels and the transmitted symbols, for each relaying protocol. A modified version of the AF receiver of [31, 32] is also proposed to enhance the performance of channel estimation. Identifiability is discussed for each PARAFAC model, and a comparative complexity analysis is made for the proposed receivers and decoders.

The rest of the paper is organized as follows. In Section 2, we present two new ST coding schemes, named MKRST and MKronST. SVD-based methods are proposed for decoding in parallel each symbol matrix. An iterative closed-form decoding solution is also presented in the Appendix. Section 3 describes the proposed MIMO relay system using the new ST codings both at the source and the relay, and PARAFAC models are established for the tensors of signals received at the relay and the destination, considering three different relaying protocols. In Section 4, we derive closed-form semi-blind receivers for each protocol. Section 5 presents Monte Carlo simulation results to illustrate the performance of the proposed receivers. Section 6 contains the conclusion and some perspectives for future work. 
Notation: Scalars, column vectors, matrices and tensors are denoted by lower-case, boldface lower-case, boldface upper-case, and calligraphic letters, e.g., $a, \mathbf{a}, \mathbf{A}, \mathcal{A}$, respectively. $\mathbf{A}_{i \text {. }}$ and $\mathbf{A}_{. j}$ represent the $i$-th row and the $j$-th column of $\mathbf{A} \in \mathbb{C}^{I \times J}$, respectively. The operator $\operatorname{diag}(\cdot)$ forms a diagonal matrix from its vector argument. The Kruskal-rank ( $k$-rank) of $\mathbf{A}$, denoted by $k_{\mathbf{A}}$, is the greatest integer $k_{\mathbf{A}}$ such that every set of $k_{\mathbf{A}}$ columns of $\mathbf{A}$ is linearly independent. The Kronecker, Khatri-Rao (column-wise Kronecker) and Hadamard products are denoted by $\otimes, \diamond$ and $*$, respectively. We use the superscripts ${ }^{T},{ }^{*},{ }^{H},{ }^{-1},{ }^{\dagger}$ for matrix transposition, complex conjugation, Hermitian transposition, inversion, and Moore-Penrose pseudo inversion, respectively.

Given a third-order tensor $\mathcal{X} \in \mathbb{C}^{I \times J \times K}$, with entries $x_{i, j, k}$, the matrices $\mathbf{X}_{J K \times I}, \mathbf{X}_{K I \times J}$, and $\mathbf{X}_{I J \times K}$ denote tall 1-mode, 2-mode and 3-mode unfoldings, with $x_{i, j, k}=\left[\mathbf{X}_{I J \times K}\right]_{(i-1) J+j, k}=\left[\mathbf{X}_{J K \times I}\right]_{(j-1) K+k, i}=$ $\left[\mathbf{X}_{K I \times J}\right]_{(k-1) I+i, j}$. A PARAFAC decomposition of $\mathcal{X} \in \mathbb{C}^{I \times J \times K}$, with rank- $R$ and matrix factors $(\mathbf{A}, \mathbf{B}, \mathbf{C})$, will be noted $\|\mathbf{A}, \mathbf{B}, \mathbf{C} ; R\|$. Tall and flat 1-mode matrix unfoldings of $\mathcal{X}$ are respectively given by

$$
\mathbf{X}_{J K \times I}=(\mathbf{B} \diamond \mathbf{C}) \mathbf{A}^{T}=\left(\mathbf{X}_{I \times J K}\right)^{T} .
$$

2-Mode and 3-mode unfoldings are easily deduced by permuting the dimensions $(I, J, K)$ and the factors $(\mathbf{A}, \mathbf{B}, \mathbf{C})$ accordingly.

\section{Space-time coding based on multiple Khatri-Rao and Kronecker products of symbol matrices}

In [8], a broad class of space time (ST) codes, called Khatri-Rao space-time (KRST) codes, is proposed for a point-to-point MIMO system. This KRST coding is composed of a linear constellation pre-coding, and a linear post-coding for time spreading. This coding has a design flexibility, spanning the range from full diversity to full transmission rate. Its main drawback is the complexity of decoding which is carried out by means of sphere decoding, as suggested in [8]. To simplify the decoding step, KRST coding without pre-coding was recently used in the context of MIMO relaying systems, at both the source and relay nodes, [31] and [32].

In this paper, we propose a new ST coding approach that generalizes the KRST scheme, while providing additional diversity in one-way two-hop MIMO relaying systems. The idea is to mutually spread in time-domain or in space-time domain several sets of data streams, by resorting to multiple Khatri-Rao or Kronecker products between symbol matrices. In the next subsection, after a brief recall of the simplified KRST coding used in [31] and [32], the so-called MKRST and MKronST encoding techniques are introduced. Parallel non-iterative decoding methods are presented for estimating each symbol matrix. Iterative decoding techniques for these multiple Khatri-Rao and Kronecker products based codes are also presented in the Appendix.

\subsection{MKRST and MKronST encoding techniques}

Consider the symbol matrix $\mathbf{S} \in \mathbb{C}^{N \times M_{s}}$ containing $N$ data-streams composed of $M_{s}$ symbols each, to be multiplexed by $M_{s}$ transmit antennas. A simplified KRST coding consists of a time spreading of the symbol matrix by means of a code matrix $\mathbf{C} \in \mathbb{C}^{P \times M_{s}}$, where $P$ is the spreading length. The encoded symbols are given by the following Khatri-Rao product

$$
\mathbf{V}=\mathbf{C} \diamond \mathbf{S} \in \mathbb{C}^{P N \times M_{s}}
$$


In scalar form, the coding is such that

$$
v_{(p-1) N+n, m_{s}}=c_{p, m_{s}} s_{n, m_{s}}
$$

showing that each symbol $s_{n, m_{s}}$ is repeated $P$ times.

In this paper, the code matrix will be chosen as a truncated discrete Fourier transform (DFT) matrix with entries $c_{p, m_{s}}=\frac{1}{\sqrt{P}} \exp \left(\frac{i 2 \pi\left(m_{s}-1\right)(p-1)}{P}\right), m_{s}=1, \cdots, M_{s} ; p=1, \cdots, P$, and $i^{2}=-1$. The matrix $\mathbf{C}$ has orthonormal columns, i.e., $\mathbf{C}^{T} \mathbf{C}^{*}=\mathbf{I}_{M_{s}}$.

Consider the matrix $\mathbf{S}$ built as multiple Khatri-Rao products of $Q \geq 2$ symbol matrices $\mathbf{S}_{q} \in \mathbb{C}^{N_{q} \times M_{s}}$ with $q=1, \cdots, Q$, i.e., $\mathbf{S}=\mathbf{S}_{1} \diamond \cdots \diamond \mathbf{S}_{q} \diamond \cdots \diamond \mathbf{S}_{Q}=\underset{q=1}{Q} \mathbf{S}_{q} \in \mathbb{C}^{N \times M_{s}}$, with $N=\prod_{q=1}^{Q} N_{q}$. The MKRST codes are given by

$$
\mathbf{V}=\mathbf{C} \diamond\left(\underset{q=1}{\stackrel{Q}{\diamond}} \mathbf{S}_{q}\right)
$$

We assume that the first row of $\mathbf{S}_{q}$, for $q=1, \cdots, Q$, is formed with ones to eliminate scaling ambiguities at decoding.

Different from the KRST code of [8], the MKRST coding induces a double time spreading of each symbol $s_{n_{q}, m_{s}}$ of the sub-matrix $\mathbf{S}_{q}$. One is due to the code matrix $\mathbf{C}$, and the other one to the multiple Khatri-Rao products with the sub-matrices $\mathbf{S}_{q^{\prime}}, q^{\prime}=1, \cdots, Q ; q^{\prime} \neq q$. The time diversity provided by each sub-matrix $\mathbf{S}_{q^{\prime}}$ is controlled by the parameter $N_{q^{\prime}}$. The encoded symbols can be written in scalar form as

$$
v_{(p-1) N+n, m_{s}}=c_{p, m_{s}} \prod_{q=1}^{Q} s_{n_{q}, m_{s}}
$$

with $n=\left(n_{1}-1\right) \prod_{q=2}^{Q} N_{q}+\left(n_{2}-1\right) \prod_{q=3}^{Q} N_{q}+\cdots+\left(n_{Q-1}-1\right) N_{Q}+n_{Q}$.

Therefore, each symbol $s_{n_{q}, m_{s}}$ of $\mathbf{S}_{q}$ is repeated $P\left(\prod_{\substack{q^{\prime}=1 \\ q^{\prime} \neq q}}^{Q} N_{q^{\prime}}\right)$ times. The main difference between KRST and MKRST codes is that, with the last one, we have supplementary design parameters to control the diversity gain. Increasing the dimensions $N_{q}$ implies an increase of time diversity, at the cost of a lower transmission rate given by $R_{\mathrm{MKRST}}=\frac{M_{s} \sum_{q=1}^{Q} N_{q}}{P \prod_{q=1}^{Q} N_{q}}$. Moreover, since the symbol matrices may have different time spans $\left(N_{q}\right)$, the time spreading (coding gain) can vary from one symbol matrix to another, allowing an unequal error protection. In a multiuser scenario, each symbol matrix $\mathbf{S}$ can be viewed as a set of data streams $\mathbf{S}_{q}$ associated with different users $(q=1, \cdots, Q)$.

We now define the MKronST coding based-on the Kronecker product of $Q \geq 2$ symbol matrices $\mathbf{S}_{q} \in$ $\mathbb{C}^{N_{q} \times M_{s_{q}}}$ with $q=1, \cdots, Q$, i.e., $\mathbf{S}=\mathbf{S}_{1} \otimes \cdots \otimes \mathbf{S}_{q} \otimes \cdots \otimes \mathbf{S}_{Q}=\otimes_{q=1}^{Q} \mathbf{S}_{q} \in \mathbb{C}^{N \times M_{s}}$, with $N=\prod_{q=1}^{Q} N_{q}$ and $M_{s}=\prod_{q=1}^{Q} M_{s_{q}}$, which implies different space-time redundancies for each symbol matrix. Eq. (4) then becomes

$$
\mathbf{V}=\mathbf{C} \diamond\left(\underset{q=1}{Q} \mathbf{S}_{q}\right)
$$


With the MKronST coding, we also assume the first row of $\mathbf{S}_{q}$ formed with ones to eliminate scaling ambiguity at decoding. This coding combines a double time spreading with one space spreading for each symbol $s_{n_{q}, m_{s_{q}}}$ of the sub-matrix $\mathbf{S}_{q} \in \mathbb{C}^{N_{q} \times M_{s_{q}}}$ which is repeated $P\left(\prod_{\substack{\prod^{\prime}=1 \\ q^{\prime} \neq q}}^{Q} N_{q^{\prime}}\right)\left(\prod_{\substack{\prod^{\prime \prime}=1 \\ q^{\prime \prime} \neq q}}^{Q} M_{s_{q^{\prime \prime}}}\right)$ times, the two products corresponding to time and space spreadings introduced by the multiple Kronecker products. For the MKronST coding, the transmission rate is given by $R_{\mathrm{MKronST}}=\frac{\sum_{q=1}^{Q} N_{q} M_{s_{q}}}{P \prod_{q=1}^{Q} N_{q}}$.

\subsection{Decoding techniques}

Once the matrix $\mathbf{S}$ estimated, the decoding consists in estimating the sub-matrices $\mathbf{S}_{q} \in \mathbb{C}^{N_{q} \times M_{s}}$ (or $\mathbf{S}_{q} \in$ $\mathbb{C}^{N_{q} \times M_{s_{q}}}$ ), with $q=1, \cdots, Q$, such that $\hat{\mathbf{S}}=\underset{q=1}{Q} \mathbf{S}_{q}\left(\right.$ or $\left.\hat{\mathbf{S}}=\underset{q=1}{\otimes} \mathbf{S}_{q}\right)$. This problem can be solved iteratively by determining the symbol matrices $\mathbf{S}_{q}$ with a two-by-two search, as described in the Appendix. This basic algorithm was proposed in [33] for estimating two matrix factors of a Khatri-Rao product associated with a third-order PARAFAC model.

A drawback of this iterative algorithm is that its performance degrades when $Q$ increases, due to error propagation. To overcome this problem, we propose a decoding procedure which operates in a parallel way for estimating each symbol matrix. Before presenting this procedure, let us recall the following formula for permuting the matrix factors $\left(\mathbf{A} \in \mathbb{C}^{I \times R}, \mathbf{B} \in \mathbb{C}^{J \times S}\right)$ of a Kronecker product

$$
\mathbf{A} \otimes \mathbf{B}=\boldsymbol{\Pi}_{I, J}(\mathbf{B} \otimes \mathbf{A}) \boldsymbol{\Pi}_{S, R}
$$

where $\Pi_{I, J}$ and $\Pi_{S, R}$ are two permutation matrices of dimensions $(I J \times J I)$ and $(S R \times R S)$, respectively, defined as

$$
\begin{aligned}
\boldsymbol{\Pi}_{I, J} & =\sum_{i} \sum_{j}\left(\mathbf{e}_{i}^{(I)} \mathbf{e}_{j}^{(J)^{T}}\right) \otimes\left(\mathbf{e}_{j}^{(J)} \mathbf{e}_{i}^{(I)^{T}}\right), \\
\boldsymbol{\Pi}_{S, R} & =\sum_{s} \sum_{r}\left(\mathbf{e}_{s}^{(S)} \mathbf{e}_{r}^{(R)^{T}}\right) \otimes\left(\mathbf{e}_{r}^{(R)} \mathbf{e}_{s}^{(S)^{S}}\right),
\end{aligned}
$$

$\mathbf{e}_{j}^{(J)}$ being the $j$-th canonical basis vector of the Euclidean space $\mathbb{R}^{J}$.

To illustrate the proposed decoding procedure, consider the case $Q=4$, with $\mathbf{S}=\mathbf{S}_{1} \otimes \mathbf{S}_{2} \otimes \mathbf{S}_{3} \otimes \mathbf{S}_{4}$. The matrices $\mathbf{S}_{1}$ and $\mathbf{S}_{4}$ can be estimated by applying the algorithm in the Appendix to the following two decompositions of $\mathbf{S}$

$$
\begin{aligned}
& \mathbf{S}=\mathbf{S}_{1} \otimes \mathbf{S}_{(2,3,4)} \text { with } \mathbf{S}_{(2,3,4)}=\mathbf{S}_{2} \otimes \mathbf{S}_{3} \otimes \mathbf{S}_{4} \\
& \mathbf{S}=\mathbf{S}_{(1,2,3)} \otimes \mathbf{S}_{4} \text { with } \mathbf{S}_{(1,2,3)}=\mathbf{S}_{1} \otimes \mathbf{S}_{2} \otimes \mathbf{S}_{3} .
\end{aligned}
$$


For estimating $\mathbf{S}_{2}$, we use the following equation obtained by permuting the factors $\mathbf{S}_{1}$ and $\mathbf{S}_{2}$

$$
\begin{aligned}
\mathbf{S}_{2} \otimes \mathbf{S}_{1} \otimes \mathbf{S}_{3} \otimes \mathbf{S}_{4} & =\boldsymbol{\Pi}_{N_{2}, N_{1}}\left(\mathbf{S}_{1} \otimes \mathbf{S}_{2}\right) \\
& =\underbrace{\left(\boldsymbol{\Pi}_{N_{2}, N_{1}} \otimes \mathbf{I}_{N_{3} N_{4}}\right)}_{\boldsymbol{\Pi}_{2}^{\text {row }}-\text { row permutation }} \mathbf{S} \underbrace{\left(\boldsymbol{\Pi}_{M_{s_{1}}, M_{s_{2}}} \otimes \mathbf{S}_{3} \otimes \mathbf{S}_{4}\right.}_{\boldsymbol{\Pi}_{2}^{\text {column }}-\text { column permutation }} \\
& =\mathbf{S}_{2} \otimes \mathbf{S}_{(1 ; 3,4)} \quad \text { with } \mathbf{S}_{(1 ; 3,4)}=\mathbf{S}_{1} \otimes \mathbf{S}_{3} \otimes \mathbf{S}_{4}
\end{aligned}
$$

Applying the algorithm in the Appendix allows to estimate $\mathbf{S}_{2}$ and $\mathbf{S}_{(1 ; 3,4)}$. Similarly, by permuting $\mathbf{S}_{3}$ with $\mathbf{S}_{1} \otimes \mathbf{S}_{2}$, we obtain

$$
\begin{aligned}
\mathbf{S}_{3} \otimes \mathbf{S}_{1} \otimes \mathbf{S}_{2} \otimes \mathbf{S}_{4} & =\underbrace{\left(\boldsymbol{\Pi}_{N_{3}, N_{1} N_{2}} \otimes \mathbf{I}_{N_{4}}\right)}_{\boldsymbol{\Pi}_{3}^{\text {row }}} \mathbf{S} \underbrace{\left(\boldsymbol{\Pi}_{M_{s_{1}} M_{s_{2}}, M_{s_{3}}} \otimes \mathbf{I}_{M_{S_{4}}}\right)}_{\boldsymbol{\Pi}_{3}^{\text {column }}} \\
& =\mathbf{S}_{3} \otimes \mathbf{S}_{(1,2 ; 4)} .
\end{aligned}
$$

In summary, for $Q=4$, we can use Eqs. (10), (12), (13) and (11) to estimate $\mathbf{S}_{1}, \mathbf{S}_{2}, \mathbf{S}_{3}$ and $\mathbf{S}_{4}$, in parallel. Such an approach can be generalized to any $Q$. Each symbol matrix $\mathbf{S}_{q}$ is estimated by applying the algorithm in the Appendix to the following equation

$$
\mathbf{S}_{q} \otimes \mathbf{S}_{1} \cdots \otimes \mathbf{S}_{q-1} \otimes \mathbf{S}_{q+1} \otimes \cdots \otimes \mathbf{S}_{Q}=\mathbf{\Pi}_{q}^{\text {row }} \mathbf{S} \boldsymbol{\Pi}_{q}^{\text {column }}
$$

where

$$
\begin{aligned}
\boldsymbol{\Pi}_{q}^{\text {row }} & =\boldsymbol{\Pi}_{N_{q}, N_{1} \cdots N_{q-1}} \otimes \mathbf{I}_{N_{q+1} \cdots N_{Q}}, \\
\boldsymbol{\Pi}_{q}^{\text {column }} & =\boldsymbol{\Pi}_{M_{s_{1}} \cdots M_{s_{q-1}}, M_{s_{q}}} \otimes \mathbf{I}_{M_{s_{q+1}} \cdots M_{s_{Q}}} .
\end{aligned}
$$

Besides avoiding error propagation, another advantage of this approach is that the estimation of the symbol matrices $\mathbf{S}_{q}, q=1, \cdots, Q$, can be parallelized. In the case of the MKRST coding where $\mathbf{S}=\mathbf{S}_{1} \diamond \cdots \diamond \mathbf{S}_{q} \diamond \cdots \diamond \mathbf{S}_{Q}$, the same procedure can be applied without column permutation.

\section{System Model}

We consider a one-way two-hop MIMO relay system, with the proposed MKRST and MKronST codings both at the source and relay nodes. The system is illustrated by means of Figure 1 where $M_{s}, M_{r}$ and $M_{d}$ denote the numbers of antennas at the source, relay, and destination nodes, with $\left(M_{s}, M_{r}, M_{d}\right) \geq 2$. The source-relay and relay-destination channels, $\mathbf{H}^{(s r)} \in \mathbb{C}^{M_{r} \times M_{s}}$ and $\mathbf{H}^{(r d)} \in \mathbb{C}^{M_{d} \times M_{r}}$, are assumed to be Rayleigh flat-fading and quasi-static during the transmission protocol. Let $\tilde{\mathcal{X}}=\mathcal{X}+\mathcal{N}$ be the noisy received signal tensor, the entries of the noise tensor $\mathcal{N}$ being zero-mean circularly-symmetric complex-valued Gaussian random variables.

During the first hop, the source transmits the MKRST or MKronST encoded symbols defined in Eqs. (4) and (6), respectively. The signals received at the relay are given by

$$
\tilde{\mathbf{X}}_{M_{r} \times P N}^{(s r)}=\mathbf{H}^{(s r)}(\mathbf{C} \diamond \mathbf{S})^{T}+\mathbf{N}_{M_{r} \times P N}^{(r)},
$$




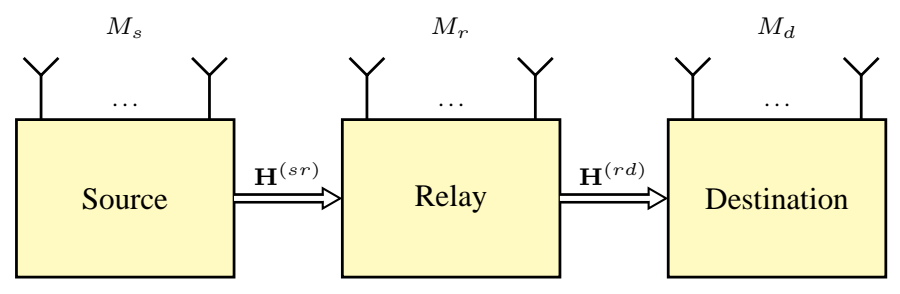

Figure 1: System Model.

where $\mathbf{N}_{M_{r} \times P N}^{(r)}$ is the noise term added at the relay.

These signals define a third-order tensor $\tilde{\mathcal{X}}^{(s r)} \in \mathbb{C}^{M_{r} \times P \times N}$ which satisfies a PARAFAC model

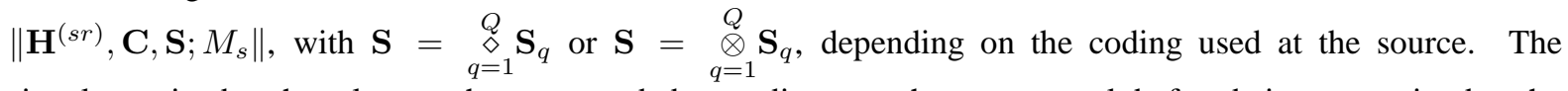
signals received at the relay are then re-encoded according to a chosen protocol, before being transmitted to the destination. In this paper, we consider three different protocols, denoted as AF, DF and EF, which are described in the next two subsections.

\subsection{AF Protocol}

With the AF protocol, the signals received at the relay are re-encoded using a simplified KRST coding with a truncated DFT matrix $\mathbf{G} \in \mathbb{C}^{J \times M_{r}}$, where $J$ is the spreading length of the relay code. This matrix satisfies also the column orthonormality property $\mathbf{G}^{T} \mathbf{G}^{*}=\mathbf{I}_{M_{r}}$ as the source code matrix.

After transmission through the relay-destination channel $\mathbf{H}^{(r d)}$, the signals received at the destination define a fourth-order tensor $\tilde{\mathcal{X}}^{(s r d)} \in \mathbb{C}^{M_{d} \times J \times P \times N}$ such as

$$
\begin{aligned}
\tilde{\mathbf{X}}_{M_{d} \times J P N}^{(s r d)} & =\mathbf{H}^{(r d)}\left(\mathbf{G} \diamond \tilde{\mathbf{X}}_{P N \times M_{r}}^{(s r)}\right)^{T}+\mathbf{N}_{M_{d} \times J P N}^{(d)}, \\
& =\mathbf{H}^{(r d)}\left(\mathbf{G} \diamond(\mathbf{C} \diamond \mathbf{S})\left(\mathbf{H}^{(s r)}\right)^{T}\right)^{T}+\mathbf{H}^{(r d)}\left(\mathbf{G} \diamond \mathbf{N}_{P N \times M_{r}}^{(r)}\right)^{T}+\mathbf{N}_{M_{d} \times J P N}^{(d)},
\end{aligned}
$$

where $\mathbf{N}_{M_{d} \times J P N}^{(d)}$ is the noise term at the destination.

As shown in [31], Eq. (19) represents a flat 1-mode unfolding of the tensor $\tilde{\mathcal{X}}^{(s r d)}$ which satisfies a nested PARAFAC model with the following factor matrices $\left(\mathbf{H}^{(r d)}, \mathbf{G}, \mathbf{H}^{(s r)}, \mathbf{C}, \mathbf{S}\right)$. However, we can also represent the tensor $\tilde{\mathcal{X}}^{(s r d)}$ by means of two PARAFAC models obtained by combining the last two modes $(p, n)$ and the first two modes $\left(m_{d}, j\right)$, respectively. One PARAFAC model $\left\|\mathbf{H}^{(r d)}, \mathbf{G}, \tilde{\mathbf{X}}_{P N \times M_{r}}^{(s r)} ; M_{r}\right\|$ is directly deduced from Eq. (18). The second PARAFAC model $\left\|\mathbf{H}_{J M_{d} \times M_{s}}^{(s r d)}, \mathbf{C}, \mathbf{S} ; M_{s}\right\|$ can be obtained by rewriting (18) as the following unfolding, with the use of 17

$$
\begin{aligned}
\tilde{\mathbf{X}}_{J M_{d} \times P N}^{(s r d)} & =\left(\mathbf{G} \diamond \mathbf{H}^{(r d)}\right) \tilde{\mathbf{X}}_{M_{r} \times P N}^{(s r)}+\mathbf{N}_{J M_{d} \times P N}^{(d)}, \\
& =\left(\mathbf{G} \diamond \mathbf{H}^{(r d)}\right) \mathbf{H}^{(s r)}(\mathbf{C} \diamond \mathbf{S})^{T}+\left(\mathbf{G} \diamond \mathbf{H}^{(r d)}\right) \mathbf{N}_{M_{r} \times P N}^{(r)}+\mathbf{N}_{J M_{d} \times P N}^{(d)}, \\
& =\mathbf{H}_{J M_{d} \times M_{s}}^{(s r d)}(\mathbf{C} \diamond \mathbf{S})^{T}+\mathbf{N}_{J M_{d} \times P N}^{(r d)}+\mathbf{N}_{J M_{d} \times P N}^{(d)},
\end{aligned}
$$

where $\mathbf{N}_{J M_{d} \times P N}^{(r d)}=\left(\mathbf{G} \diamond \mathbf{H}^{(r d)}\right) \mathbf{N}_{M_{r} \times P N}^{(r)}$ represents the noise added at relay received at destination, and

$$
\mathbf{H}_{J M_{d} \times M_{s}}^{(s r d)}=\left(\mathbf{G} \diamond \mathbf{H}^{(r d)}\right) \mathbf{H}^{(s r)},
$$


Table 1: Noiseless PARAFAC models of received signals tensors, with the protocols AF, DF and EF. For EF, replace $\hat{\hat{\mathbf{S}}}$ by $\hat{\mathbf{S}}$.

\begin{tabular}{|c|c|}
\hline $\mathrm{AF}$ & $\mathrm{DF} / \mathrm{EF}$ \\
\hline $\mathbf{X}_{J M_{d} \times P N}^{(s r d)}=\mathbf{H}_{J M_{d} \times M_{s}}^{(s r d)}(\mathbf{C} \diamond \mathbf{S})^{T}$ & $\mathbf{X}_{M_{r} \times P N}^{(s r)}=\mathbf{H}^{(s r)}(\mathbf{C} \diamond \mathbf{S})^{T}$ \\
\hline $\mathbf{X}_{J M_{d} \times P N}^{(s r d)}=\left(\mathbf{G} \diamond \mathbf{H}^{(r d)}\right) \mathbf{X}_{M_{r} \times P N}^{(s r)}$ & $\mathbf{X}_{M_{d} \times J N}^{(r d)}=\mathbf{H}^{(r d)}(\mathbf{G} \diamond \hat{\hat{\mathbf{S}}})^{T}$ \\
\hline
\end{tabular}

can be interpreted as a tall 3-mode unfolding of the effective channel tensor $\mathcal{H}^{(s r d)} \in \mathbb{C}^{M_{d} \times J \times M_{s}}$ between the source and the destination.

The transmission rate of the proposed relaying system is dependent on the coding used. For the MKRST coding, the source transmits $M_{s} \sum_{q=1}^{Q} N_{q}$ symbols in $P \prod_{q=1}^{Q} N_{q}$ symbol periods. Then, the relay takes $J P \prod_{q=1}^{Q} N_{q}$ symbol periods for forwarding the symbols to the destination, during the second hop. That gives the following transmission rate

$$
R_{\mathrm{MKRST}}^{\mathrm{AF}}=\frac{M_{s} \sum_{q=1}^{Q} N_{q}}{P(J+1) \prod_{q=1}^{Q} N_{q}} .
$$

In the case of the MKronST coding, it is easy to derive the following transmission rate

$$
R_{\mathrm{MKronST}}^{\mathrm{AF}}=\frac{\sum_{q=1}^{Q} N_{q} M_{s_{q}}}{P(J+1) \prod_{q=1}^{Q} N_{q}} .
$$

Comparing (25) with (24), one can conclude that the transmission rate of the MKRST coding is higher than the one of the MKronST coding for which $M_{s}=\prod_{q=1}^{Q} M_{s_{q}}$. However, this last coding provides more redundancies of transmitted symbols due to the space diversity introduced by multiple Kronecker products.

\subsection{DF and EF Protocols}

In this subsection, we consider the case where the relay and the source have the same number of antennas $\left(M_{r}=M_{s}\right)$. With the DF protocol, the matrix $\hat{\mathbf{S}}$ estimated at the relay is decoded by applying the parallel decoding described in section 2.2 and then the estimated symbols are projected onto the alphabet. That gives estimated symbol matrices $\hat{\mathbf{S}}_{q}, q=1, \cdots, Q$, which are re-encoded using the MKRST (or MKronST) coding as $\hat{\hat{\mathbf{S}}}=\underset{q=1}{\stackrel{Q}{\&}} \hat{\mathbf{S}}_{q}$ (or $\hat{\mathbf{S}}=\underset{q=1}{Q} \hat{\mathbf{S}}_{q}$ ). In the case of the EF protocol, the estimated matrix $\hat{\mathbf{S}}$ is directly re-encoded using the relay code matrix $\mathbf{G} \in \mathbb{C}^{J \times M_{s}}$. 
After transmission through the relay-destination channel $\mathbf{H}^{(r d)}$, the signals received at destination are given by

$$
\tilde{\mathbf{X}}_{M_{d} \times J N}^{(r d)}=\mathbf{H}^{(r d)}(\mathbf{G} \diamond \hat{\mathbf{S}})^{T}+\mathbf{N}_{M_{d} \times J N}^{(d)}
$$

for the DF protocol, and

$$
\tilde{\mathbf{X}}_{M_{d} \times J N}^{(r d)}=\mathbf{H}^{(r d)}(\mathbf{G} \diamond \hat{\mathbf{S}})^{T}+\mathbf{N}_{M_{d} \times J N}^{(d)}
$$

for the EF protocol.

The third-order tensor $\tilde{\mathcal{X}}^{(r d)} \in \mathbb{C}^{M_{d} \times J \times N}$ containing the signals received by the $M_{d}$ destination antennas in $J N$ symbol periods, satisfies the PARAFAC model $\| \mathbf{H}^{(r d)}, \mathbf{G}, \hat{\mathbf{S}}$ or $\hat{\hat{\mathbf{S}}} ; M_{s} \|$. Table 1 summarizes the PARAFAC models for each relaying protocol.

The transmission rates for the MKRST and MKronST coding-based systems using the DF protocol are given by

$$
R_{\mathrm{MKRST}}^{\mathrm{DF}}=\frac{M_{s} \sum_{q=1}^{Q} N_{q}}{(P+J) \prod_{q=1}^{Q} N_{q}} .
$$

and

$$
R_{\mathrm{MKronST}}^{\mathrm{DF}}=\frac{\sum_{q=1}^{Q} N_{q} M_{s_{q}}}{(P+J) \prod_{q=1}^{Q} N_{q}} .
$$

We have to note that, as for the AF protocol, the transmission rate with the MKRST coding is higher than the one with the MKronST coding. Moreover, the EF protocol leads to the same transmission rates as the DF protocol, since the only difference between them is in the decoding and not in the transmission. In conclusion, the DF and EF protocols are more efficient in terms of transmission rate than the AF one. Finally, the transmission rates for the simplified KRST coding used in [31] and [32] can be obtained as particular cases of (24) and (28) leading to the following expressions $R_{\mathrm{KRST}}^{\mathrm{AF}}=\frac{M_{s}}{P(J+1)}$, and $R_{\mathrm{KRST}}^{\mathrm{DF}}=\frac{M_{s}}{P+J}$.

In the next section, we present closed-form semi-blind receivers that jointly estimate the two-hop channels and the transmitted symbols for the MKRST and MKronST codings, in distinguishing the three considered protocols. We present necessary identifiability conditions and scaling ambiguity relations for the tensor models. A comparative complexity analysis is also made for the proposed semi-blind receivers.

\section{Closed-Form Semi-Blind Receivers}

Assume the code matrices $\mathbf{C}$ and $\mathbf{G}$ have a truncated DFT structure and are known at the relay and destination nodes. We derive closed-form receivers for jointly estimating the individual channels $\left(\mathbf{H}^{(s r)}, \mathbf{H}^{(r d)}\right)$ and the transmitted symbols $\left(\mathbf{S}_{q}, q=1, \cdots, Q\right)$. For each protocol, we first present the Khatri-Rao products which are used by the receivers at the relay and destination nodes. 


\subsection{Semi-Blind Receiver for the AF Protocol}

The transmitted symbol matrix $\mathbf{S}$ can be estimated at the destination using the following tall 3-mode unfolding of $\tilde{\mathcal{X}}^{(s r d)}$ deduced from (22) by permuting the matrix factors

$$
\tilde{\mathbf{X}}_{J M_{d} N \times P}^{(s r d)}=\left(\mathbf{H}_{J M_{d} \times M_{s}}^{(s r d)} \diamond \mathbf{S}\right) \mathbf{C}^{T}+\mathbf{N}_{J M_{d} N \times P}^{(r d)}+\mathbf{N}_{J M_{d} N \times P}^{(d)} .
$$

The source code matrix $\mathbf{C}$ being assumed column-orthonormal $\left(\mathbf{C}^{T} \mathbf{C}^{*}=\mathbf{I}_{M_{s}}\right)$, which implies $P \geq M_{s}$, a least square (LS) estimate of the Khatri-Rao product $\mathbf{R}=\mathbf{H}_{J M_{d} \times M_{s}}^{(s r d)} \diamond \mathbf{S}$ is given by

$$
\hat{\mathbf{R}}=\tilde{\mathbf{X}}_{J M_{d} N \times P}^{(s r d)} \mathbf{C}^{*} \in \mathbb{C}^{J M_{d} N \times M_{s}} .
$$

Once $\mathbf{R}$ estimated, the factor matrices $\left(\mathbf{H}_{J M_{d} \times M_{s}}^{(s r d)}, \mathbf{S}\right)$ of the Khatri-Rao product can be obtained by applying the algorithm described in the Appendix. Then, the symbol matrices $\mathbf{S}_{q}, q=1, \cdots, Q$, are decoded from the estimate $\hat{\mathbf{S}}$ by applying the parallel decoding algorithm described in section 2.2

The channel $\mathbf{H}^{(r d)}$ can be estimated at the destination using the following tall 2-mode unfolding of $\tilde{\mathcal{X}}^{(s r d)}$ deduced from (20)

$$
\tilde{\mathbf{X}}_{M_{d} P N \times J}^{(s r d)}=\left(\mathbf{H}^{(r d)} \diamond \tilde{\mathbf{X}}_{P N \times M_{r}}^{(s r)}\right) \mathbf{G}^{T}+\mathbf{N}_{M_{d} P N \times J}^{(d)}
$$

Owing to the column orthonormality of the code matrix $\mathbf{G}$, the LS estimate of the Khatri-Rao product $\mathbf{Y}=$ $\mathbf{H}^{(r d)} \diamond \tilde{\mathbf{X}}_{P N \times M_{r}}^{(s r)}$ is given by

$$
\hat{\mathbf{Y}}=\tilde{\mathbf{X}}_{M_{d} P N \times J}^{(s r d)} \mathbf{G}^{*} \in \mathbb{C}^{M_{d} P N \times M_{r}} .
$$

Once $\mathbf{Y}$ estimated, the factors $\left(\mathbf{H}^{(r d)}, \tilde{\mathbf{X}}_{P N \times M_{s}}^{(s r)}\right.$ ) of the Khatri-Rao product can be obtained by applying the algorithm described in the Appendix.

To estimate the channel $\mathbf{H}^{(s r)}$, we use the transpose of Eq. (17), with $\mathbf{S}$ and $\tilde{\mathbf{X}}_{P N \times M_{r}}^{(s r)}$ replaced by their estimated values obtained in previous steps of the algorithm. The LS estimation gives

$$
\hat{\mathbf{H}}^{(s r) T}=(\mathbf{C} \diamond \hat{\mathbf{S}})^{\dagger} \hat{\mathbf{X}}_{P N \times M_{r}}^{(s r)}
$$

Furthermore, exploiting the orthonormality property of $\mathbf{C}$ simplifies this estimation as

$$
\begin{aligned}
\hat{\mathbf{H}}^{(s r) T} & =\left(\left(\mathbf{C}^{H} \mathbf{C}\right) *\left(\hat{\mathbf{S}}^{H} \hat{\mathbf{S}}\right)\right)^{-1}(\mathbf{C} \diamond \hat{\mathbf{S}})^{H} \hat{\mathbf{X}}_{P N \times M_{r}}^{(s r)} \\
& =\operatorname{diag}^{-1}\left(\rho_{1}, \cdots, \rho_{M_{s}}\right)(\mathbf{C} \diamond \hat{\mathbf{S}})^{H} \hat{\mathbf{X}}_{P N \times M_{r}}^{(s r)}
\end{aligned}
$$

where $\rho_{m_{s}}=\left\|\hat{\mathbf{S}}_{m_{s}}\right\|_{2}^{2}, m_{s}=1, \cdots, M_{s}$.

The same approach can be used to estimate $\mathbf{H}^{(s r)}$ from $(23)$, with $\mathbf{H}^{(r d)}$ and $\mathbf{H}_{J M_{d} \times M_{s}}^{(s r d)}$ replaced by their estimated values obtained in previous steps of the algorithm, as

$$
\hat{\mathbf{H}}^{(s r)}=\operatorname{diag}^{-1}\left(\varrho_{1}, \cdots, \varrho_{M_{r}}\right)\left(\mathbf{G} \diamond \hat{\mathbf{H}}^{(r d)}\right)^{H} \hat{\mathbf{H}}_{J M_{d} \times M_{s}}^{(s r d)},
$$

with $\varrho_{m_{r}}=\left\|\hat{\mathbf{H}}_{. m_{r}}^{(r d)}\right\|_{2}^{2}, m_{r}=1, \cdots, M_{r}$.

The closed-form receiver for the AF protocol, denoted as AF-KRF, is summarized in Table 3. Note that in [32] it was also proposed an AF-KRF receiver. However, in [32] the channel matrices $\mathbf{H}^{(r d)}$ and $\mathbf{H}^{(s r)}$ are estimated from the 2-mode unfolding $\mathbf{H}_{M_{d} M_{s} \times J}^{(s r d)}=\left(\mathbf{H}^{(r d)} \diamond \mathbf{H}^{(s r) T}\right) \mathbf{G}^{T}$ deduced from (23) and estimated from (30). It is 
worth noting that, contrary to [32], the proposed AF-KRF receiver directly estimates the relay-destination channel $\mathbf{H}^{(r d)}$ from the received signal tensor $\tilde{\mathcal{X}}^{(s r d)}$. As will be shown later in the simulation results (c.f. Fig. (4), this new solution provides a significant performance improvement in terms of normalized mean square error (NMSE), in comparison with the solution of [32].

\subsection{Semi-Blind Receiver for the DF and EF Protocols}

Differently from the AF-KRF receiver for which the estimation is carried out at the destination only, in the DF and EF cases the estimation is split between the relay and the destination.

With the DF protocol, the symbol matrix $\mathbf{S}$ and the channel $\mathbf{H}^{(s r)}$ are first estimated at the relay using the tall 2-mode unfolding of $\tilde{\mathcal{X}}^{(s r)}$ deduced from the PARAFAC model $\left\|\mathbf{H}^{(s r)}, \mathbf{C}, \mathbf{S} ; M_{s}\right\|$

$$
\tilde{\mathbf{X}}_{M_{s} N \times P}^{(s r)}=\left(\mathbf{H}^{(s r)} \diamond \mathbf{S}\right) \mathbf{C}^{T}+\mathbf{N}_{M_{s} N \times P}^{(d)}
$$

Exploiting the orthonormality property of $\mathbf{C}$ gives the following LS estimate of the Khatri-Rao product $\mathbf{Z}=$ $\mathbf{H}^{(s r)} \diamond \mathbf{S}$

$$
\hat{\mathbf{Z}}=\tilde{\mathbf{X}}_{M_{s} N \times P}^{(s r)} \mathbf{C}^{*} \in \mathbb{C}^{M_{s} N \times M_{s}} .
$$

Then, the symbol matrix $\mathbf{S}$ and the channel $\mathbf{H}^{(s r)}$ are estimated from $\hat{\mathbf{Z}}$ by applying the algorithm in the Appendix.

Once the matrix $\mathbf{S}$ estimated, the sub-matrices $\mathbf{S}_{q}, q=1, \cdots, Q$, are estimated using the decoding approach of subsection 2.2, and the estimated symbols are projected onto the alphabet.

Then, a new symbol matrix denoted as $\hat{\hat{\mathbf{S}}}$ is calculated using the estimated matrices $\hat{\mathbf{S}}_{q}$ formed with the projected symbols, and it is re-encoded with the relay code matrix $\mathbf{G}$. The flat 1-mode unfolding (26) of the tensor $\tilde{\mathcal{X}}^{(r d)} \in \mathbb{C}^{M_{d} \times J \times N}$ containing the signals received at destination can be rearranged as the following tall 2-mode unfolding

$$
\tilde{\mathbf{X}}_{M_{d} N \times J}^{(r d)}=\left(\mathbf{H}^{(r d)} \diamond \hat{\hat{\mathbf{S}}}\right) \mathbf{G}^{T}+\mathbf{N}_{M_{d} N \times J}^{(d)} .
$$

The LS estimate of the Khatri-Rao product $\mathbf{Q}=\mathbf{H}^{(r d)} \diamond \hat{\hat{\mathbf{S}}}$ is given by

$$
\hat{\mathbf{Q}}=\tilde{\mathbf{X}}_{M_{d} N \times J}^{(r d)} \mathbf{G}^{*} \in \mathbb{C}^{M_{d} N \times M_{s}} .
$$

Applying the algorithm in the Appendix allows to estimate the factors $\left(\mathbf{H}^{(r d)}, \hat{\mathbf{S}}\right)$ from the estimate $\hat{\mathbf{Q}}$. Then, the same procedure as the one used at the relay allows to estimate the symbols from the estimate $\hat{\hat{\mathbf{S}}}$.

In the case of the EF protocol, the matrix $\hat{\mathbf{S}}$ estimated at the relay is directly re-encoded as shown by Eq. (27), i.e., without decoding and projection onto the alphabet. That implies a lower computational complexity than the one with the DF protocol, at the cost of a possible performance degradation. The channel $\mathbf{H}^{(r d)}$ and the matrix $\mathbf{S}$ are then estimated from Eqs. (39) and (40) with $\hat{\hat{\mathbf{S}}}$ replaced by $\hat{\mathbf{S}}$.

Table 2 summarizes the Khatri-Rao products considered for estimating the symbols and channels matrices.

\subsection{Identifiability and scaling ambiguity relations}

The system parameter identifiability is linked to the uniqueness of the LS estimates of the Khatri-Rao products $\mathbf{R}, \mathbf{Y}, \mathbf{Z}$ and $\mathbf{Q}$, i.e. the full column rank property of the code matrices $\mathbf{C}$ and $\mathbf{G}$ inducing the uniqueness of the right inverse of $\mathbf{C}^{T}$ and $\mathbf{G}^{T}$ in Eqs. (30), (32), (37) and (39). That implies the necessary conditions $P \geq M_{s}$ and 
Table 2: Khatri-Rao products used by the receivers with the protocols AF, DF and EF. For EF, replace $\hat{\hat{\mathbf{S}}}$ by $\hat{\mathbf{S}}$.

\begin{tabular}{|c|c|}
\hline $\mathrm{AF}$ & $\mathrm{DF} / \mathrm{EF}$ \\
\hline$\tilde{\mathbf{X}}_{J M_{d} N \times P}^{(s r d)} \mathbf{C}^{*}=\mathbf{H}_{J M_{d} \times M_{s}}^{(s r d)} \diamond \mathbf{S}$ & $\tilde{\mathbf{X}}_{M_{r} N \times P}^{(s r)} \mathbf{C}^{*}=\mathbf{H}^{(s r)} \diamond \mathbf{S}$ \\
\hline$\tilde{\mathbf{X}}_{M_{d} P N \times J}^{(s r d)} \mathbf{G}^{*}=\mathbf{H}^{(r d)} \diamond \tilde{\mathbf{X}}_{P N \times M_{r}}^{(s r)}$ & $\tilde{\mathbf{X}}_{M_{d} N \times J}^{(r d)} \mathbf{G}^{*}=\mathbf{H}^{(r d)} \diamond \hat{\hat{\mathbf{S}}}$ \\
\hline
\end{tabular}

$J \geq M_{r}$. Note that the choice of a truncated DFT structure for $\mathbf{C}$ and $\mathbf{G}$ allows to simplify the calculation of the pseudo-inverses in Eqs. (35) and (36).

Disregarding the noise, the estimated matrices $\mathbf{H}^{(s r)}, \mathbf{H}^{(r d)}$ and $\mathbf{S}$ are equal to their true values up to column scaling ambiguities (permutation ambiguity does not exist due to the knowledge of $\mathbf{C}$ and $\mathbf{G}$ ). For eliminating these scaling ambiguities, we assume the first row of each symbol matrix $\mathbf{S}_{q}, q=1, \cdots, Q$, is equal to an all-ones vector. This assumption is necessary for symbol decoding, regardless of the considered protocol. Then, the final estimates of the channels and symbols matrices for the $\mathrm{AF}$ receiver are obtained as

$$
\begin{array}{r}
\hat{\mathbf{S}} \leftarrow \hat{\mathbf{S}} \boldsymbol{\Lambda}_{\mathbf{S}}, \quad \hat{\mathbf{H}}_{J M_{d} \times M_{s}}^{(s r d)} \leftarrow \hat{\mathbf{H}}_{J M_{d} \times M_{s}}^{(s r d)}\left(\boldsymbol{\Lambda}_{\mathbf{S}}\right)^{-1}, \\
\hat{\mathbf{H}}^{(r d)} \leftarrow \hat{\mathbf{H}}^{(r d)} \boldsymbol{\Lambda}_{\mathbf{H}^{(r d)}}, \quad \hat{\mathbf{X}}_{P N \times M_{r}}^{(s r)} \leftarrow \hat{\mathbf{X}}_{P N \times M_{r}}^{(s r)}\left(\boldsymbol{\Lambda}_{\left.\mathbf{H}^{(r d)}\right)^{-1}},\right.
\end{array}
$$

where $\boldsymbol{\Lambda}_{\mathbf{S}}=\operatorname{diag}^{-1}\left(\hat{\mathbf{S}}_{1 .}\right)$ and $\boldsymbol{\Lambda}_{\mathbf{H}^{(r d)}}=\operatorname{diag}^{-1}\left(\hat{\mathbf{H}}_{1 .}^{(r d)}\right) \operatorname{diag}\left(\mathbf{H}_{1 .}^{(r d)}\right)$.

In the case of the DF receiver, the final estimates are given by

$$
\begin{aligned}
\hat{\mathbf{S}} \leftarrow \hat{\mathbf{S}} \boldsymbol{\Lambda}_{\mathbf{S}}, \quad \hat{\mathbf{H}}^{(s r)} & \leftarrow \hat{\mathbf{H}}^{(s r)}\left(\boldsymbol{\Lambda}_{\mathbf{S}}\right)^{-1}, \\
\hat{\mathbf{H}}^{(r d)} \leftarrow \hat{\mathbf{H}}^{(r d)} \boldsymbol{\Lambda}_{\mathbf{H}^{(r d)}}, \quad \hat{\hat{\mathbf{S}}} & \leftarrow \hat{\hat{\mathbf{S}}}\left(\boldsymbol{\Lambda}_{\mathbf{H}^{(r d)}}\right)^{-1} .
\end{aligned}
$$

Note that the first rows of $\mathbf{H}^{(r d)}$ and $\mathbf{S}$ are assumed to be known at the destination. In practice, the first row of $\mathbf{H}^{(r d)}$ can be estimated using a short training sequence generated at the relay as proposed in [32].

The closed-form receivers associated with the DF and EF protocols, denoted as DF-KRF and EF-KRF respectively, are summarized in Table 3 .

\subsection{Complexity Analysis}

In this section, a comparison of the computational complexity of the proposed closed-form semi-blind receivers is provided. The dominant complexity cost is associated with the SVD-based rank-one approximations to compute the factors of the Khatri-Rao matrix products using the algorithm in the Appendix. Note that, for a matrix of dimensions $J \times K$, the complexity of its $\operatorname{SVD}$ is $\mathbb{O}(\min (J, K) J K)$ [37].

For the AF-KRF receiver, the computational complexity associated with joint channel and symbol estimation is concentrated at the destination node, whereas for the DF-KRF receiver, the complexity is shared between the relay and destination nodes.

The AF-KRF receiver calculates $M_{s}$ and $M_{r}$ SVD-based rank-one approximations in steps (1.2) and (1.7) of Table 3 , respectively. The computational cost of step (1.2) is $M_{s} \mathbb{O}\left(\min \left(J M_{d}, N\right) J M_{d} N\right)$, the factor $M_{s}$ coming 
1. Joint estimation of $\mathbf{H}^{(s r)}, \mathbf{H}^{(r d)}$ and $\mathbf{S}$.

(A) AF-KRF receiver

Given $\tilde{\mathcal{X}}^{(s r d)}, \mathbf{C}$ and $\mathbf{G}$.

(1.1) Compute the LS estimate of $\mathbf{R}=\mathbf{H}_{J M_{d} \times M_{s}}^{(s r d)} \diamond \mathbf{S}$ using 31 .

(1.2) Use the algorithm in the Appendix to estimate $\mathbf{H}_{J M_{d} \times M_{s}}^{(s r d)}$ and $\mathbf{S}$ from $\hat{\mathbf{R}}$.

(1.3) Remove the scaling ambiguities of $\hat{\mathbf{S}}$ and $\hat{\mathbf{H}}_{J M_{d} \times M_{s}}^{(s r d)}$ using 41 .

(1.4) Use the parallel decoding algorithm of subsection 2.2 to estimate the symbol matrices $\mathbf{S}_{q} ; q=1, \cdots, Q$ from $\hat{\mathbf{S}}$.

(1.5) Project the estimated symbols onto the alphabet.

(1.6) Compute the LS estimate of $\mathbf{Y}=\mathbf{H}^{(r d)} \diamond \tilde{\mathbf{X}}_{P N \times M_{r}}^{(s r)}$ using 33 .

(1.7) Use the algorithm in the Appendix to estimate $\mathbf{H}^{(r d)}$ and $\tilde{\mathbf{X}}_{P N \times M_{r}}^{(s r)}$ from $\hat{\mathbf{Y}}$.

(1.8) Remove the scaling ambiguities of $\hat{\mathbf{H}}^{(r d)}$ and $\hat{\mathbf{X}}^{(s r)}$ using 42.

(1.9) Compute the LS estimate of $\mathbf{H}^{(s r)}$ using 35] or 36.

(B) DF-KRF receiver

Given $\tilde{\mathcal{X}}^{(s r)}, \tilde{\mathcal{X}}^{(r d)}, \mathbf{C}$ and $\mathbf{G}$.

- Relay Processing

(1.1) Compute the LS estimate of $\mathbf{Z}=\mathbf{H}^{(s r)} \diamond \mathbf{S}$ using 38 .

(1.2) Use the algorithm in the Appendix to estimate $\mathbf{H}^{(s r)}$ and $\mathbf{S}$ from $\hat{\mathbf{Z}}$

(1.3) Remove the scaling ambiguities of $\hat{\mathbf{S}}$ and $\hat{\mathbf{H}}^{(s r)}$ using 43].

(1.4) Use the parallel decoding algorithm of subsection 2.2 to estimate the symbol matrices $\mathbf{S}_{q} ; q=1, \cdots, Q$ from $\hat{\mathbf{S}}$.

(1.5) Project the estimated symbols onto the alphabet.

(1.6) Compute $\hat{\hat{\mathbf{S}}}=\underset{q=1}{\stackrel{Q}{\stackrel{\mathbf{S}}{q}}} \hat{\mathbf{A}}_{q}$, or $\hat{\hat{\mathbf{S}}}=\underset{q=1}{\otimes} \hat{\mathbf{S}}_{q}$.

(1.7) Re-encode $\hat{\hat{\mathbf{S}}}$ using $\mathbf{G}$.

- Destination Processing

(1.8) Compute the LS estimate of $\mathbf{Q}=\mathbf{H}^{(r d)} \diamond \hat{\hat{\mathbf{S}}}$ using 40].

(1.9) Use the algorithm in the Appendix to estimate $\mathbf{H}^{(r d)}$ and $\hat{\mathbf{S}}$ from $\hat{\mathbf{Q}}$.

(1.10) Remove the scaling ambiguities of $\hat{\hat{\mathbf{S}}}$ and $\hat{\mathbf{H}}^{(r d)}$ using 44).

(1.11) Use the parallel decoding algorithm of subsection 2.2 to estimate the symbol matrices $\mathbf{S}_{q}$.

(1.12) Project the estimated symbols onto the alphabet.

(C) EF-KRF receiver

Given $\tilde{\boldsymbol{\mathcal { X }}}^{(s r)}, \tilde{\boldsymbol{\mathcal { X }}}^{(r d)}, \mathbf{C}$ and $\mathbf{G}$.

Steps (1.1)-(1.2) of the DF-KRF receiver.

Steps (1.7)-(1.12) of the DF-KRF receiver, with $\hat{\mathbf{S}}$ replaced by $\hat{\mathbf{S}}$. 
Table 4: Complexities of the receivers with the AF, DF and EF protocols, and of MKRST and MKronST decodings.

\begin{tabular}{|c|c|r|}
\hline \multirow{2}{*}{ Receivers } & AF-KRF & $M_{s} \mathbb{O}\left(\min \left(J M_{d}, N\right) J M_{d} N\right)+M_{r} \mathbb{O}\left(\min \left(M_{d}, P N\right) M_{d} P N\right)$ \\
\cline { 2 - 4 } & $\mathrm{DF} / \mathrm{EF}-\mathrm{KRF}$ & $M_{s} \mathbb{O}\left(\min \left(M_{s}, N\right) M_{s} N\right)+M_{s} \mathbb{O}\left(\min \left(M_{d}, N\right) M_{d} N\right)$ \\
\hline \multirow{2}{*}{ Decoders } & MKRST & $\mathbb{O}\left(\sum_{q=1}^{Q-1} N_{q} \min \left(N_{q}, \prod_{q^{\prime}=q+1}^{Q} N_{q^{\prime}}\right) \prod_{q^{\prime}=q+1}^{Q} N_{q^{\prime}} M_{s}\right)$ \\
\cline { 2 - 4 } & MKronST & $\mathbb{O}\left(\sum_{q=1}^{Q-1} N_{q} M_{s_{q}} \min \left(N_{q} M_{s_{q}}, \prod_{q^{\prime}=q+1}^{Q} N_{q^{\prime}} M_{s_{q^{\prime}}}\right) \cdot \prod_{q^{\prime}=q+1}^{Q} N_{q^{\prime}} M_{s_{q^{\prime}}}\right)$ \\
\hline
\end{tabular}

from the fact that the calculation of the matrix factors of the Khatri-Rao product is made column-by-column. For step (1.7), the computational cost is $M_{r} \mathbb{O}\left(\min \left(M_{d}, P N\right) M_{d} P N\right)$.

For the DF-KRF receiver $\left(M_{r}=M_{s}\right)$, the complexity to estimate the Khatri-Rao product factors from $\hat{\mathbf{Z}}$ is given by $M_{s} \mathbb{O}\left(\min \left(M_{s}, N\right) M_{s} N\right)$, while the complexity associated with the processing at destination, i.e. step (1.9) in Table 3 , is $M_{s} \mathbb{O}\left(\min \left(M_{d}, N\right) M_{d} N\right)$.

To estimate the $\mathbf{S}_{q} \in \mathbb{C}^{N_{q} \times M_{s}}$ matrices from their estimated Khatri-Rao product, we need to perform $(Q-$ 1) $M_{s}$ SVDs. In Table 4 the complexity is presented for each receiver and for both decoders. Since $\hat{\mathbf{S}}$ is not reconstructed at the relay for the EF-KRF receiver, the complexity of the MKRST (or MKronST) decoding is absent for the EF-KRF.

Note that MKronST requires to compute SVDs of matrices with larger dimensions than for MKRST, for $q=1, \cdots, Q-1$. The computation time associated with the permutation of symbol matrices for the decoding algorithm, as presented in Section 2.2, can be considered negligible.

\section{Simulation Results}

In this section, simulation results are provided to evaluate the performance of the proposed MKRST and MKronST coding schemes and semi-blind receivers, with the objective to show: i) the effectiveness of the parallel MKRST decoding algorithm proposed in Section 2.2 for three different values of $Q$ (Fig. 2), ii) the symbol error rate (SER) and the channels NMSE performances of the proposed semi-blind receivers associated with each relaying protocol for the MKRST coding (Figs. 3 and 4), iii) the time and space diversity gains provided by MKRST and MKronST coding schemes with the DF-KRF receiver (Figs. 5, 6, and 7, iv) the impact of the spreading parameters on the SER performance of individual symbol matrices, with the DF-KRF receiver for the MKronST scheme (Fig. 8), and v) the impact of the relay positioning on the SER performance for each hop (Fig. 9).

As a reference for comparison, we also consider the standard KRST coding scheme adopted in the previous works [31] and [32] for relay-assisted MIMO systems. Two performance criteria are considered, namely, the SER 
Table 5: Values of design parameters used in the simulations.

\begin{tabular}{|c|c|}
\hline Figures & Simulation Parameters \\
\hline Fig. 2 & MKRST: $M_{s_{q}}=N_{q}=2$ \\
\hline Figs. 3 and 4 & AF-KRF MKRST $Q=2: M_{s}=N_{1}=N_{2}=P=J=M_{r}=M_{d}=2$ \\
& DF/EF-KRF MKRST $Q=2: M_{s}=N_{1}=N_{2}=M_{r}=M_{d}=2, P=J=3$ \\
\hline Fig. 5 & KRST: $N=P=M_{s}=J=M_{r}=M_{d}=4$ \\
& MKRST $Q=2: N_{1}=N_{2}=2, J=M_{r}=M_{d}=P=M_{s}=4$ \\
\hline Fig. 6 & KKRST $Q=3: N_{1}=3, N_{2}=2, N_{3}=1, P=J=M_{r}=M_{d}=M_{s}=4$ \\
& MKronST $Q=2: N_{1}=N_{2}=M_{s_{1}}=M_{s_{2}}=2, P=J=M_{r}=M_{d}=4$ \\
\hline Fig. 7 & MKRST $Q=2: N_{1}=N_{2}=M_{r}=M_{s}=2, M_{d}=P=J=4$ \\
\hline Fig. 8 & MKronST $Q=3: N_{1}=N_{3}=M_{s_{1}}=M_{s_{2}}=2, N_{2}=M_{s_{3}}=1, P=J=M_{r}=M_{d}=4$ \\
\hline Fig. 9 & MKronST $Q=2: M_{s_{2}}=N_{1}=N_{2}=M_{s_{1}}=2, P=J=M_{r}=M_{d}=4$ \\
\hline
\end{tabular}

and the NMSE of the estimated channels, calculated as:

$$
\operatorname{NMSE}(\mathbf{H})=\frac{1}{M} \sum_{m=1}^{M} \frac{\left\|\hat{\mathbf{H}}_{m}-\mathbf{H}_{m}\right\|_{F}^{2}}{\left\|\mathbf{H}_{m}\right\|_{F}^{2}},
$$

where $\hat{\mathbf{H}}_{m}$ is the channel matrix estimated at the $m$-th run, and $M$ denotes the number of Monte Carlo runs. The SER and NMSE curves represent averages over at least $4 \times 10^{3}$ Monte Carlo runs. Each run corresponds to different realizations of the channel matrices, symbol matrices, and noise tensors. The channel matrices $\mathbf{H}^{(s r)}$ and $\mathbf{H}^{(r d)}$ have i.i.d. zero-mean complex Gaussian entries with variances $1 / M_{s}$ and $1 / M_{r}$, respectively. The transmitted symbols are randomly drawn from a 4-QAM alphabet $( \pm 1 \pm j) / \sqrt{2}$. The noise variance is assumed to be the same at the relay and the destination. The SER and NMSE curves are plotted as a function of the symbol energy to noise spectral density ratio $\left(E_{s} / N_{0}\right)$. At each run, the ratio $E_{s} / N_{0}$ is controlled by fixing $E_{s}=1$ and varying $N_{0}$ at the relay and the destination to ensure the desired $E_{s} / N_{0}$ value. The code matrices are chosen as truncated DFT matrices, so that $\mathbf{C}$ and $\mathbf{G}$ have orthonormal columns, i.e., $\mathbf{C}^{T} \mathbf{C}^{*}=\mathbf{I}_{M_{s}}$ and $\mathbf{G}^{T} \mathbf{G}^{*}=\mathbf{I}_{M_{r}}$. The design parameters of the coding schemes are chosen to ensure that all the systems simulated in a given figure have the same transmission rate. For convenience, these design parameters are provided in Table 5

In Fig. 2. we compare the proposed parallel decoding algorithm described in Section 2.2 with the iterative decoding one (c.f. Appendix). For this experiment, we choose the MKRST scheme and consider different values of $Q$. In the case $Q=2$, both decoding algorithms exhibit similar SER performances. However, as $Q$ increases the proposed parallel decoding algorithm achieves a lower SER due to the absence of error propagation, which is not the case when using the iterative decoding algorithm. This result shows the effectiveness of parallel decoding for $Q>2$.

Figure 3 compares three different relaying protocols at the relay, leading to the AF-, DF- and EF-KRF 
receivers. As a reference for comparison, we also consider an ideal version of EF-KRF, where the relay re-encodes the exact symbol matrix $\mathbf{S}$. We can see that the DF-KRF receiver performs slightly better than the EF-KRF one in a range of moderate $E_{s} / N_{0}$ values. Note also that the DF-KRF performs much better than the AF-KRF one. Indeed, the DF-KRF receiver is less sensitive to noise amplification, due to the fact that the relay forwards estimated symbols to the destination, instead of the noisy signals received at the relay. This explains the improved SER performance of the DF-KRF receiver compared with the AF-KRF one.

Figure 4 shows the NMSE of the estimated channels $\mathbf{H}^{(s r)}$ and $\mathbf{H}^{(r d)}$ obtained with the AF- and DF-based receivers, assuming the MKRST code with $Q=2$. In this experiment, we also include the performance of the AF-KRF receiver of [32]. Recall that the receiver of [32] estimates $\mathbf{H}^{(s r)}$ and $\mathbf{H}^{(r d)}$ from the effective channel $\mathbf{H}^{(s r d)}$ using (30) ad (23), while the proposed AF-KRF receiver estimates $\mathbf{H}^{(r d)}$ directly from the received signal tensor $\tilde{\mathcal{X}}^{(s r d)}$. The results show an improved performance of the proposed AF-KRF receiver over the solution provided in [32] for the estimation of $\mathbf{H}^{(r d)}$. We clearly see that DF-KRF yields more accurate estimations of both channel matrices, due to signal reconstruction at the relay. In the subsequent experiments, we restrict ourselves to the DF-KRF receiver due to its superior performance as illustrated by these results.

In Fig. 5, we compare the KRST and MKRST coding schemes under two configurations, $Q=2$ and $Q=3$. We can see that the MKRST scheme with $Q=3$ provides the best performance with a gain of $3 \mathrm{~dB}$ for a SER of $10^{-3}$, in comparison with the KRST coding scheme. The MKRST coding with $Q=3$ offers a SNR gain of 1.25 $\mathrm{dB}$ for a SER of $10^{-3}$, in comparison with the case $Q=2$. These results corroborate the coding gain provided by MKRST due to the mutual time spreading of information symbols.

In another experiment, we concentrate our attention to the MKronST coding scheme. It can bee seen from Fig. 6 that the MKronST code performs better than the KRST code in both configurations $(Q=2$ and $Q=3$ ). More specifically, for a SER of $10^{-3}$, the SNR gains of MKronST over KRST are around $2.25 \mathrm{~dB}$ and $5 \mathrm{~dB}$, respectively. These gains come from the mutual space-time spreading of the symbols provided by the MKronST code, resulting in additional diversities, as opposed to the conventional KRST code which does not have this feature.

Figure [7 compares the MKRST and MKronST schemes with $Q=2$. The performance of the MKronST is better than that of MKRST for moderate and high $E_{s} / N_{0}$ values. For a target SER of $10^{-3}$, the gain is around $3 \mathrm{~dB}$. Indeed, as explained previously, MKronST provides an extra mutual spreading of the symbols across the transmit antennas, while with MKRST the transmitted symbols are mutually spread across symbol periods only.

To illustrate the impact of the design parameters $N_{q}$ and $M_{s_{q}}$ of the MKronST code on the SER performance, we evaluate the SER individually for each $\mathbf{S}_{q}$, with $Q=3$. The symbol matrices have the following dimensions: $\mathbf{S}_{1}(2 \times 2), \mathbf{S}_{2}(3 \times 2)$ and $\mathbf{S}_{3}(2 \times 1)$. The symbols of $\mathbf{S}_{1}, \mathbf{S}_{2}$, and $\mathbf{S}_{3}$ are then repeated $12 P, 8 P$, and $24 P$ times, respectively. As shown in Fig. 8 , the SER performance of $\mathbf{S}_{3}$ is the best, followed by those of $\mathbf{S}_{1}$ and $\mathbf{S}_{2}$.

In Fig. 9 we show the impact of the relay positioning on the SER performance for each hop. We assume that the channels $\mathbf{H}^{(s r)}$ and $\mathbf{H}^{(r d)}$ have i.i.d. zero-mean circularly-symmetric complex Gaussian entries with variances $1 /\left(d_{0}^{\beta} M_{s}\right)$ and $1 /\left(\left(d-d_{0}\right)^{\beta} M_{r}\right)$, respectively, where $d$ denotes the distance between the source and destination nodes and $d_{0}$ the distance between the source and the relay. In this experiment, we assume $\beta=3$, and we consider three different cases regarding the relay positioning, $d_{0}=d / 3, d_{0}=d / 2$ and $d_{0}=2 d / 3$. The first case corresponds to a situation where the relay is placed closer to the source. In the second one, the relay is half the distance between source and relay. In the last one, the relay is placed closer to the destination. The 
simulation results show that the SER associated with the first hop (calculated at the relay after symbol decoding) improves when the relay is closer to the source, as expected. However, we can see that the SER performance at the destination is nearly the same for all the three cases. This result indicates the presence of error propagation due to decoding at the relay, which is the factor dominating the performance of the receiver at the destination.

\section{Conclusion}

We have proposed two new ST codings based on multiple Khatri-Rao and Kronecker products of symbol matrices, denoted as MKRST and MKronST, respectively. A parallel closed-form decoding algorithm has been described for estimating the transmitted symbol matrices from their Khatri-Rao or Kronecker product. Using MKRST and MKronST codings at the source and relay nodes, and exploiting the PARAFAC models for the tensors of signals received at the relay and the destination, we have derived closed-form semi-blind receivers for jointly estimating the individual channels and the symbol matrices, with three different relaying protocols (AF, DF and EF).

A computational complexity analysis of the proposed receivers has been provided, and an extensive performance evaluation has been carried out by means of Monte Carlo simulations. The simulation results have demonstrated that the new MKRST and MKronST coding schemes outperform the standard KRST coding, while supporting multiuser transmissions under different rates and code lengths per user. Another contribution of this paper concerns the use of DF and EF relaying protocols which allows to significantly improve the SER performance at the cost of a supplementary computational complexity at the relay. As perspectives to this work, we shall consider a more general multihop MIMO relaying scenario as well as the use of space-time-frequency transmission in MIMO-OFDM relaying systems.

Instead of using several rank-one SVDs to estimate the symbol matrices, higher-order methods such as the sequential rank-one approximations with projection (SeROAP) recently proposed in [38] could also be considered.

\section{Appendix}

\section{Iterative algorithm for estimating Khatri-Rao product factors}

Given the Khatri-Rao product $\mathbf{S}=\mathbf{S}_{1} \diamond \mathbf{S}_{2}$, the factor matrices $\mathbf{S}_{1} \in \mathbb{C}^{N_{1} \times M_{s}}$ and $\mathbf{S}_{2} \in \mathbb{C}^{N_{2} \times M_{s}}$ can be estimated by calculating the rank-one approximation of the matrix defined for each column $\left(m=1, \cdots, M_{s}\right)$ as

$$
\mathbf{F}_{. m}=\operatorname{unvec}_{N_{2} \times N_{1}}\left[\mathbf{S}_{. m}\right]=\left(\mathbf{S}_{2}\right) . m\left(\mathbf{S}_{1}\right)_{. m}^{T} \text {. }
$$

Defining the singular value decomposition (SVD) of $\mathbf{F}_{. m}=\mathbf{U} \boldsymbol{\Sigma} \mathbf{V}^{H}$, the $m$-th column of $\mathbf{S}_{1}$ and $\mathbf{S}_{2}$ are given by $\left(\hat{\mathbf{S}}_{1}\right)_{. m}=\sqrt{\sigma_{1}} \mathbf{V}_{.1}^{*}$ and $\left(\hat{\mathbf{S}}_{2}\right)_{. m}=\sqrt{\sigma_{1}} \mathbf{U}_{.1}$,

where $\mathbf{U}_{.1}$ and $\mathbf{V}_{.1}$ represent the first column of $\mathbf{U}$ and $\mathbf{V}$ associated with the largest singular value $\sigma_{1}$ of $\mathbf{F} . m$, respectively.

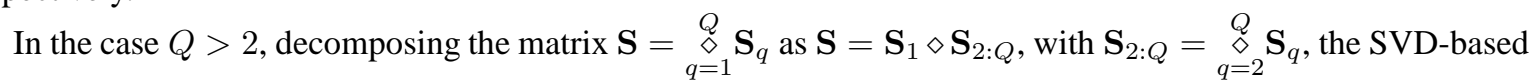
algorithm described above can be applied to estimate the factors $\mathbf{S}_{1}$ and $\mathbf{S}_{2: Q}$. Then, from the decomposition $\hat{\mathbf{S}}_{2: Q} \cong \mathbf{S}_{2} \diamond \mathbf{S}_{3: Q}$, where $\mathbf{S}_{3: Q}=\underset{q=3}{Q} \mathbf{S}_{q}$, applying the above algorithm allows to estimate the factors $\mathbf{S}_{2}$ and $\mathbf{S}_{3: Q}$. Repeating the same procedure $Q-1$ times provides an estimation of the $Q$ factors $\mathbf{S}_{q}, q=1, \cdots, Q$. 
Note that the estimates of the factor matrices $\left(\mathbf{S}_{1}, \mathbf{S}_{2}\right)$ of the Khatri-Rao product $\mathbf{S}_{1} \diamond \mathbf{S}_{2}$ are obtained up to a scalar scaling factor for each column $m_{s}=1, \cdots, M_{s}$. Therefore, to eliminate these scaling ambiguities, one needs to know one element for each column, i.e. one row of $\mathbf{S}_{1}$ or $\mathbf{S}_{2}$. In our context, since $\mathbf{S}$ is itself estimated from a Khatri-Rao product, the a priori knowledge of the first row of each $\mathbf{S}_{q}$ for $q=1 \cdots, Q$, is needed to carry out the MKRST decoding without ambiguity.

\section{References}

[1] F. H. P. Fitzek and M. D. Katz, Cooperation in Wireless Networks: Principles and Applications. Springer Netherlands, 2006.

[2] T. Cover and A. E. Gamal, "Capacity theorems for the relay channel," IEEE Transactions on Information Theory, vol. 25, no. 5, pp. 572-584, Sep 1979.

[3] A. Sendonaris, E. Erkip, and B. Aazhang, "User cooperation diversity. Part I. System description," IEEE Transactions on Communications, vol. 51, no. 11, pp. 1927-1938, Nov 2003.

[4] — - "User cooperation diversity. Part II. Implementation aspects and performance analysis," IEEE Transactions on Communications, vol. 51, no. 11, pp. 1939-1948, Nov 2003.

[5] J. N. Laneman, D. N. C. Tse, and G. W. Wornell, "Cooperative diversity in wireless networks: Efficient protocols and outage behavior," IEEE Transactions on Information Theory, vol. 50, no. 12, pp. 3062-3080, Dec 2004.

[6] N. D. Sidiropoulos, G. B. Giannakis, and R. Bro, "Blind PARAFAC receivers for DS-CDMA systems," IEEE Transactions on Signal Processing, vol. 48, no. 3, pp. 810-823, Mar 2000.

[7] R. A. Harshman, "Foundations of the PARAFAC procedure: Models and conditions for an "explanatory" multi-modal factor analysis," UCLA Working Papers in Phonetics, vol. 16, no. 1, p. 84, 1970.

[8] N. D. Sidiropoulos and R. S. Budampati, "Khatri-Rao space-time codes," IEEE Transactions on Signal Processing, vol. 50, no. 10, pp. 2396-2407, Oct 2002.

[9] A. L. F. de Almeida and W. C. Freitas, "Blind receiver for multi-layered space-frequency coded MIMO schemes based on temporally extended linear constellation precoding," in The IEEE 74th Vehicular Technology Conference (VTC Fall), Sept 2011, pp. 1-5.

[10] W. C. Freitas, A. L. F. de Almeida, and J. P. C. L. da Costa, "Blind joint channel estimation and data detection for precoded multi-layered space-frequency MIMO schemes," Circuits, Systems, and Signal Processing, vol. 33, no. 4, pp. 1215-1229, 2014.

[11] G. Favier and A. L. F. de Almeida, "Overview of constrained PARAFAC models," EURASIP Journal on Advances in Signal Processing, no. 1, pp. 1-25 Sep. 2014. 
[12] M. F. Kader and S. Y. Shin, "Cooperative relaying using space-time block coded non-orthogonal multiple access," IEEE Transactions on Vehicular Technology, vol. 66, no. 7, pp. 5894-5903, July 2017.

[13] J. He, V. Tervo, S. Qian, M. Juntti and T. Matsumoto, "Performance analysis of OSTBC transmission in lossy forward MIMO relay networks,'IEEE Communications Letters, vol. 21, no. 8, pp. 1791-1794, Aug. 2017.

[14] A. L. F. de Almeida, G. Favier, and J. C. M. Mota, "Space-time multiplexing codes: A tensor modeling approach," in The 7th IEEE Workshop on Signal Processing Advances in Wireless Communications, July 2006, pp. 1-5.

[15] A. L. F. de Almeida, G. Favier, C. C. Cavalcante, and J. C. M. Mota, "Tensor-based space-time multiplexing codes for MIMO-OFDM systems with blind detection," in The 17th IEEE International Symposium on Personal, Indoor and Mobile Radio Communications, Sept 2006, pp. 1-5.

[16] A. L. F. de Almeida, G. Favier, and J. C. M. Mota, "A constrained factor decomposition with application to MIMO antenna systems," IEEE Transactions on Signal Processing, vol. 56, no. 6, pp. 2429-2442, June 2008.

[17] A. L. F. de Almeida, G. Favier, and J. C. Mota, "Space-time spreading-multiplexing for MIMO wireless communication systems using the PARATUCK-2 tensor model," Signal Processing, vol. 89, no. 11, pp. 2103-2116, 2009.

[18] G. Favier, M. N. da Costa, A. L. F. de Almeida, and J. M. T. Romano, “Tensor space-time (TST) coding for MIMO wireless communication systems," Signal Processing, vol. 92, no. 4, pp. 1079-1092, 2012.

[19] G. Favier and A. L. F. de Almeida, "Tensor space-time-frequency coding with semi-blind receivers for MIMO wireless communication systems," IEEE Transactions on Signal Processing, vol. 62, no. 22, pp. 5987-6002, Nov 2014.

[20] A. L. F. de Almeida, G. Favier, and J. C. M. Mota, "PARAFAC-based unified tensor modeling for wireless communication systems with application to blind multiuser equalization," Signal Processing, vol. 87, no. 2, pp. 337-351, 2007.

[21] A. L. F. de Almeida and G. Favier, "Double Khatri-Rao space-time-frequency coding using semi-blind PARAFAC based receiver," IEEE Signal Processing Letters, vol. 20, no. 5, pp. 471-474, May 2013.

[22] F. Roemer and M. Haardt, "Tensor-based channel estimation and iterative refinements for two-way relaying with multiple antennas and spatial reuse," IEEE Transactions on Signal Processing, vol. 58, no. 11, pp. 5720-5735, Nov 2010.

[23] Y. Rong, M. R. A. Khandaker, "Channel estimation of dual-hop MIMO relay systems using parallel factor analysis," in The 17th Asia Pacific Conference on Communications, Oct 2011, pp. 278-283.

[24] J. Du, C. Yuan, and J. Zhang, "Low complexity PARAFAC-based channel estimation for non-regenerative MIMO relay systems," IET Communications, vol. 8, no. 12, pp. 2193-2199, August 2014. 
[25] X. Han, A. L. F. de Almeida, and Z. Yang, "Channel estimation for MIMO multi-relay systems using a tensor approach,” EURASIP Journal on Advances in Signal Processing, vol. 2014, no. 1, p. 163, 2014.

[26] I. V. Cavalcante, A. L. F. de Almeida, and M. Haardt, "Joint channel estimation for three-hop MIMO relaying systems," IEEE Signal Processing Letters, vol. 22, no. 12, pp. 2430-2434, Dec 2015.

[27] C. A. R. Fernandes, A. L. F. de Almeida, and D. B. da Costa, "Unified tensor modeling for blind receivers in multiuser uplink cooperative systems," IEEE Signal Process. Lett, vol. 19, pp. 247-250, 2012.

[28] A. L. F. de Almeida, C. A. R. Fernandes, and D. B. da Costa, "Multiuser detection for uplink DS-CDMA amplify-and-forward relaying systems," IEEE Signal Processing Letters, vol. 20, no. 7, pp. 697-700, July 2013.

[29] W. C. Freitras Jr., G. Favier, A. L. F. de Almeida, "Sequential Closed-Form Semiblind Receiver for Space-Time Coded Multihop Relaying Systems," IEEE Signal Processing Letters, vol. 24, no. 12, pp. 1773-1777, Dec. 2017.

[30] L. R. Ximenes, G. Favier, A. L. F. de Almeida, and Y. C. B. Silva, "PARAFAC-PARATUCK semi-blind receivers for two-hop cooperative MIMO relay systems," IEEE Transactions on Signal Processing, vol. 62, no. 14 , pp. 3604-3615, July 2014.

[31] L. R. Ximenes, G. Favier, and A. L. F. de Almeida, "Semi-blind receivers for non-regenerative cooperative MIMO communications based on nested PARAFAC modeling," IEEE Transactions on Signal Processing, vol. 63, no. 18 , pp. 4985-4998, Sept 2015.

[32] — - "Closed-form semi-blind receiver for MIMO relay systems using double Khatri-Rao space-time coding," IEEE Signal Processing Letters, vol. 23, no. 3, pp. 316-320, March 2016.

[33] A. Y. Kibangou and G. Favier, "Non-iterative solution for PARAFAC with a Toeplitz matrix factor," in The 17th European Signal Processing Conference, 2009, pp. 691-695.

[34] C. F. V. Loan and N. Pitsianis, Approximation with Kronecker Products. Dordrecht: Springer Netherlands, 1993, pp. 293-314.

[35] G. Favier, C. A. R. Fernandes, and A. L. de Almeida, "Nested Tucker tensor decomposition with application to MIMO relay systems using tensor space-time coding (TSTC)," Signal Processing, vol. 128, pp. 318-331, 2016.

[36] A. L. F. de Almeida, G. Favier, and L. R. Ximenes, "Space-time-frequency (STF) MIMO communication systems with blind receiver based on a generalized PARATUCK-2 model," IEEE Transactions on Signal Processing, vol. 61, no. 8, pp. 1895-1909, April 2013.

[37] G. H. Golub and C. F. V. Loan, Matrix Computations (3rd Ed.). Baltimore, MD, USA: Johns Hopkins University Press, 1996.

[38] A. P. da Silva, P. Comon, and A. L. F. de Almeida, "A finite algorithm to compute rank-1 tensor approximations," IEEE Signal Processing Letters, vol. 23, no. 7, pp. 959-963, July 2016. 


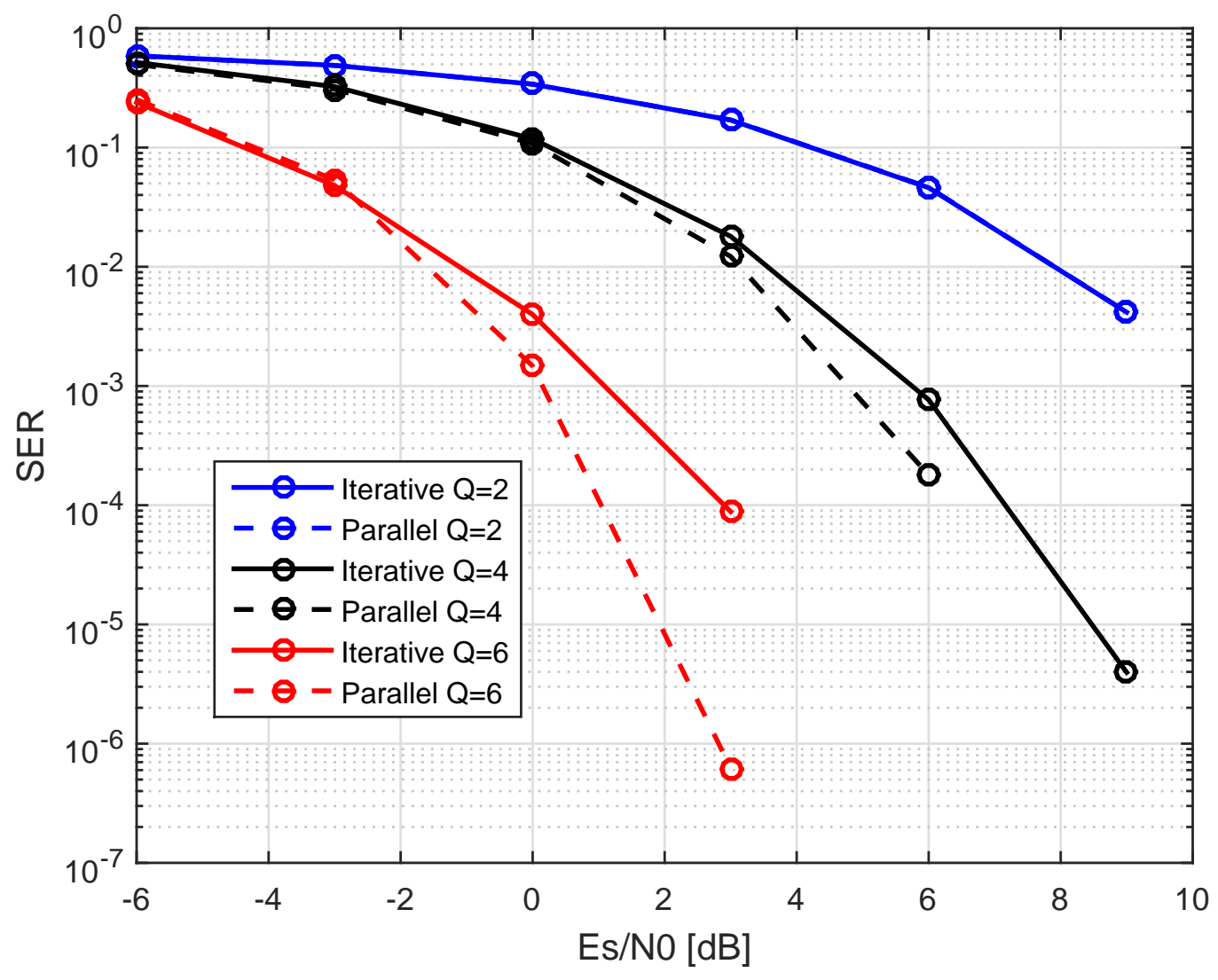

Figure 2: SER comparison with iterative and parallel decoding algorithms, for MKRST and three values of $Q$. 


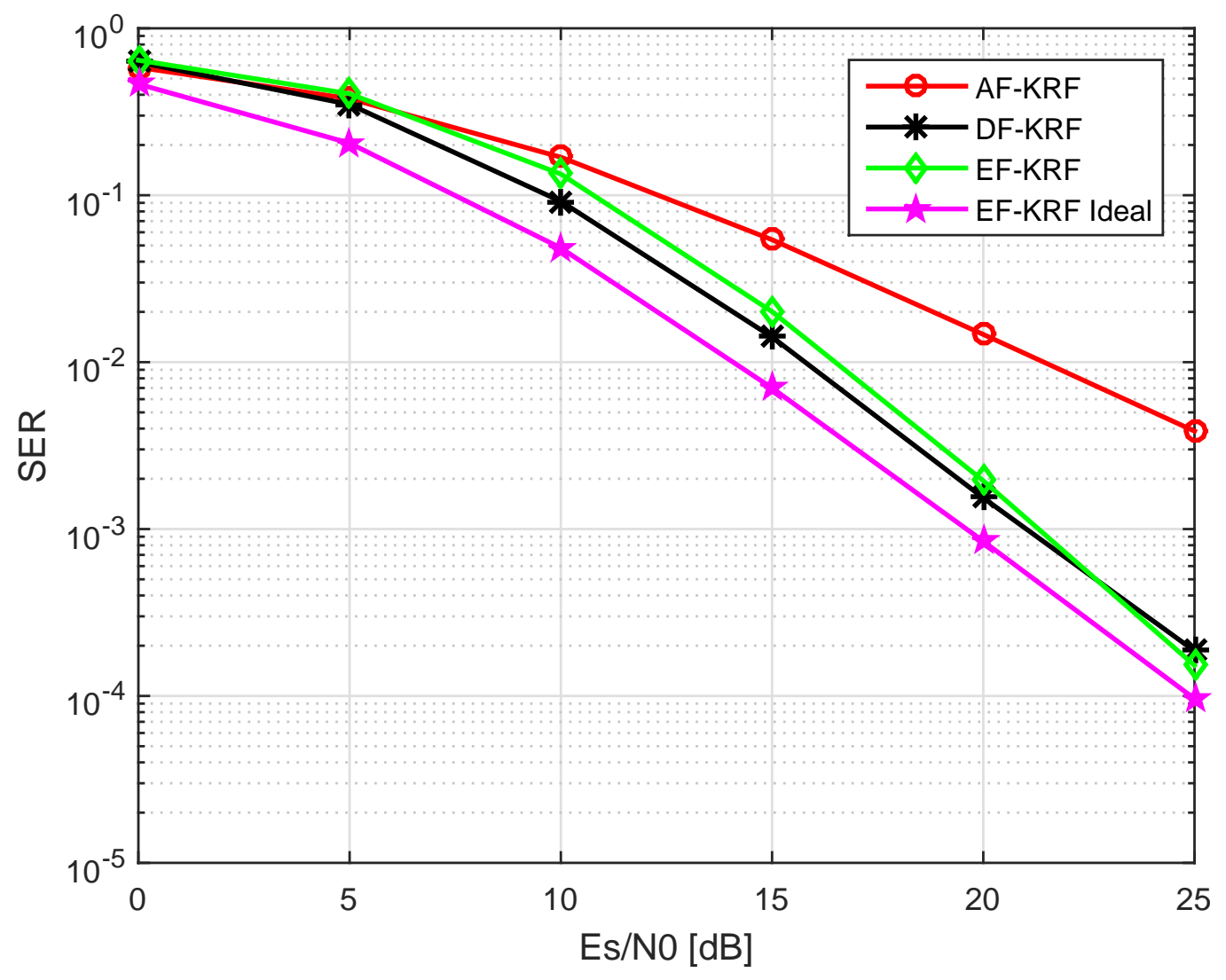

Figure 3: SER comparison between AF-KRF and DF/EF-KRF receivers for MKRST with $Q=2$. 

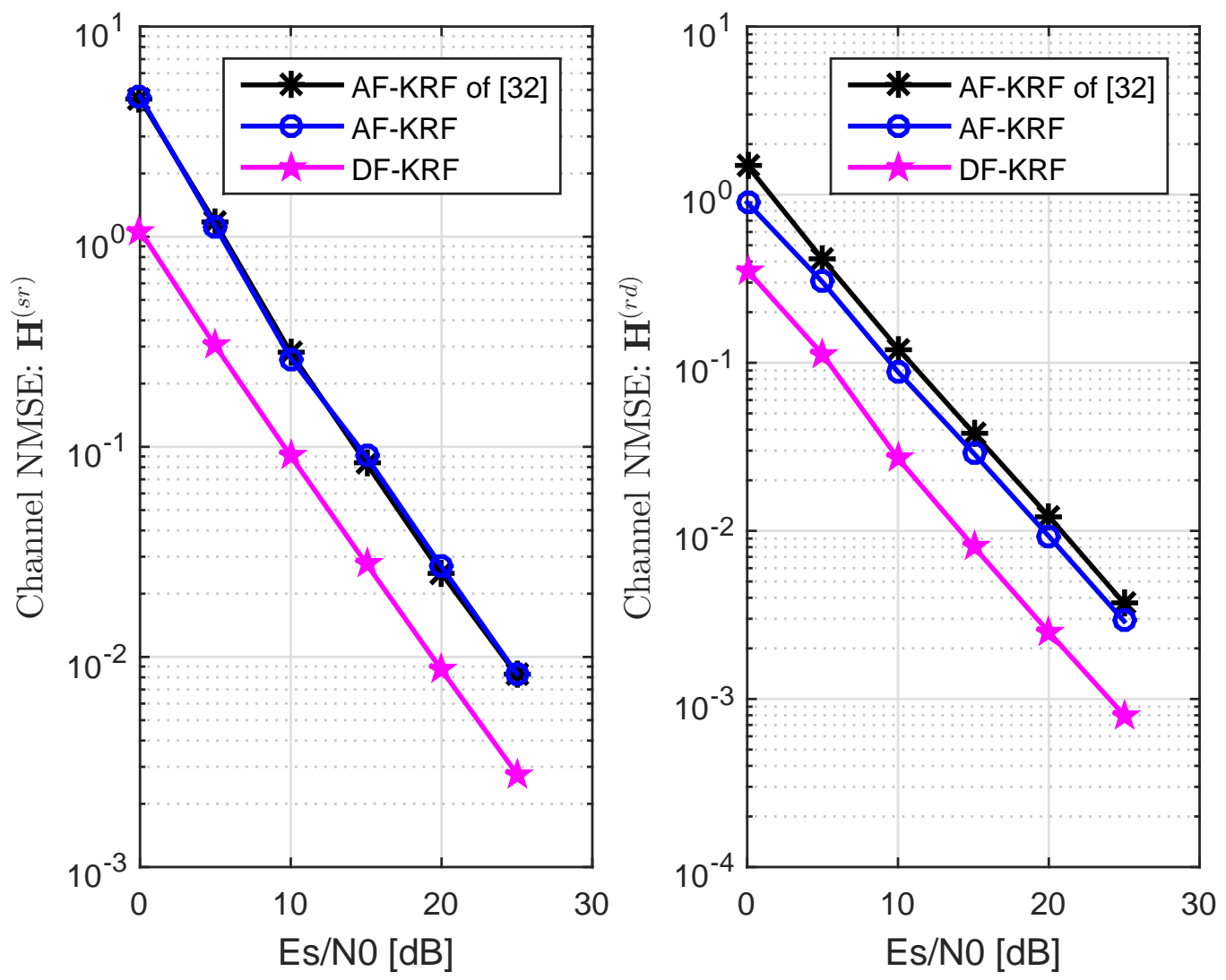

Figure 4: Channels NMSE for AF-KRF and DF-KRF receivers for MKRST with $Q=2$. 


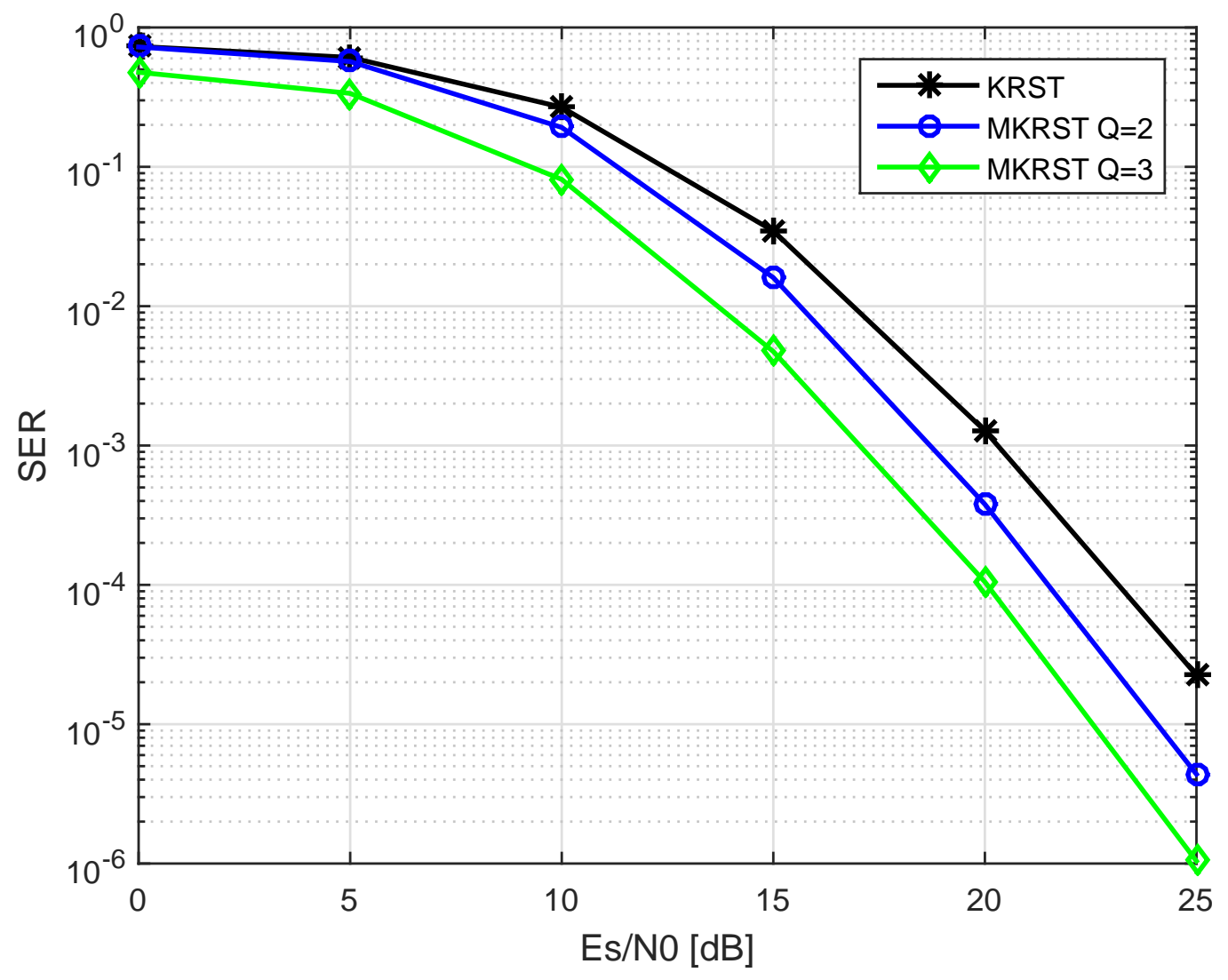

Figure 5: SER comparison between KRST and MKRST with DF-KRF receiver. 


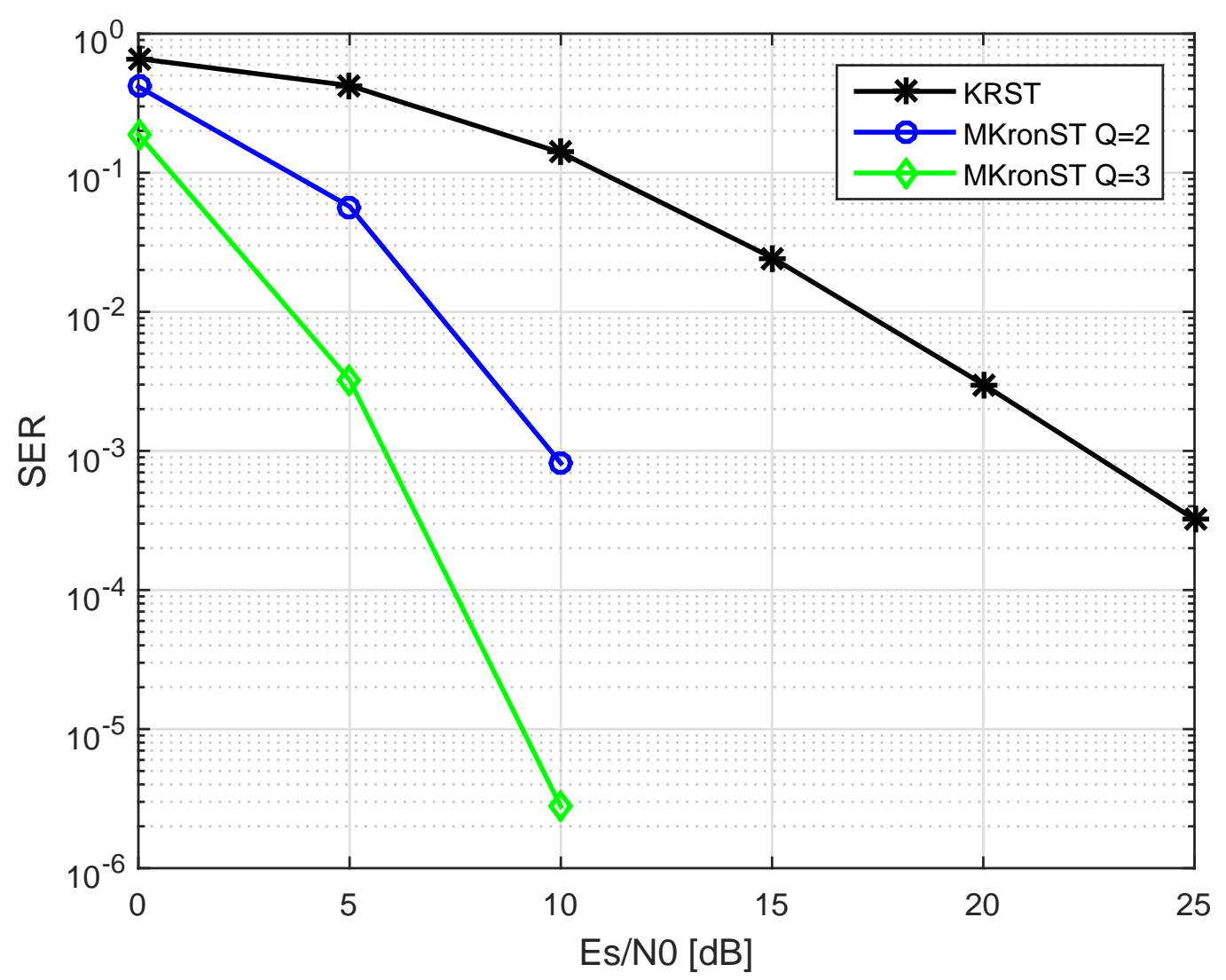

Figure 6: SER comparison between KRST and MKronST with DF-KRF receiver. 


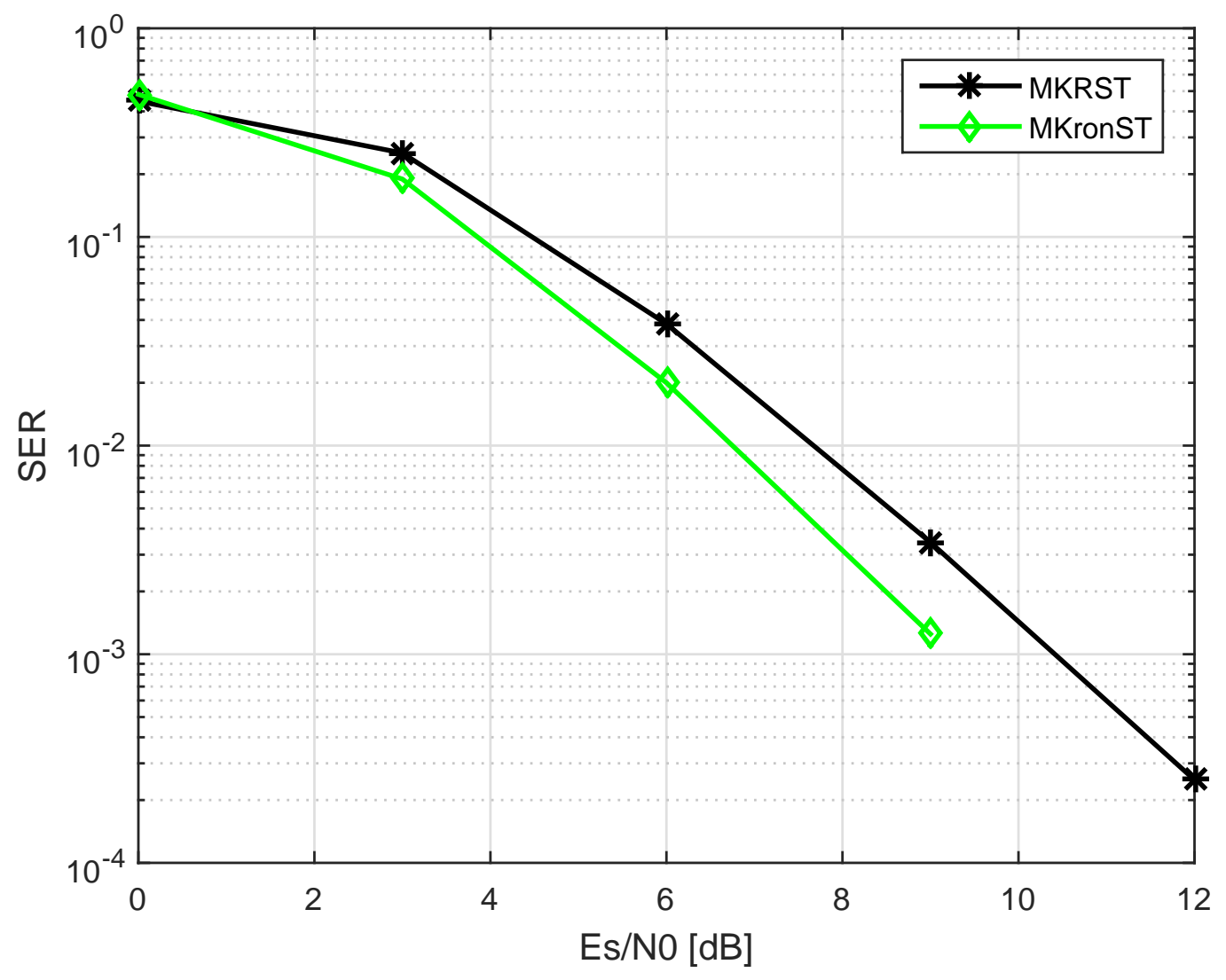

Figure 7: SER comparison between MKRST and MKronST with $\mathrm{Q}=2$ and DF-KRF receiver. 


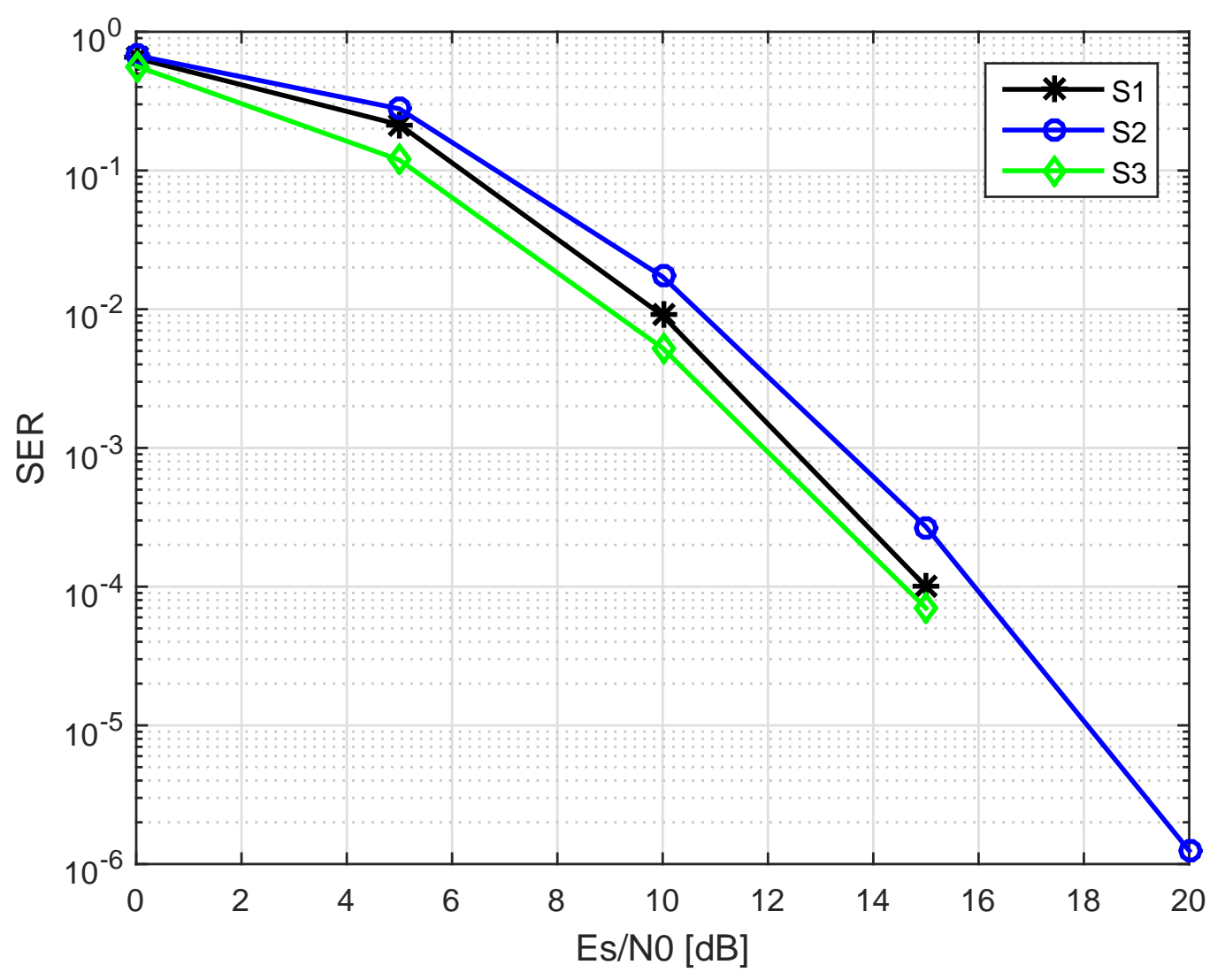

Figure 8: SER of individual symbol matrices for MKronST with $Q=3$ and DF-KRF receiver. 

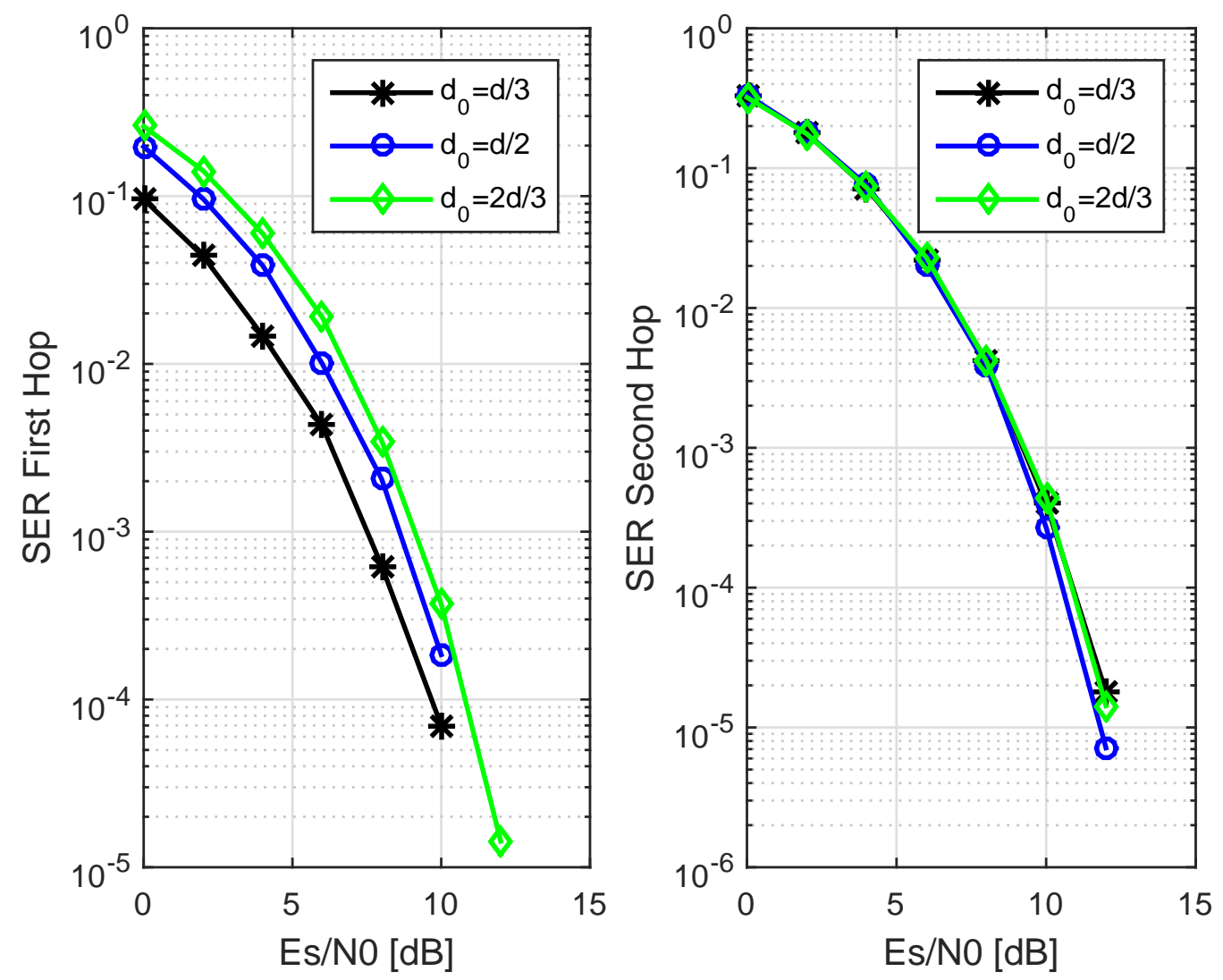

Figure 9: SER impact of relay position for MKRST with $Q=2$ and DF-KRF receiver. 